\title{
An Essential Role for RAX Homeoprotein and NOTCH-HES Signaling in Otx2 Expression in Embryonic Retinal Photoreceptor Cell Fate Determination
}

\author{
Yuki Muranishi, ${ }^{1 \star}$ Koji Terada, ${ }^{1 *}$ Tatsuya Inoue, ${ }^{1,3}$ Kimiko Katoh,,${ }^{1,2}$ Toshinori Tsujii, ${ }^{1}$ Rikako Sanuki, ${ }^{1,2}$ \\ Daisuke Kurokawa, ${ }^{4}$ Shinichi Aizawa, ${ }^{4}$ Yasuhiro Tamaki, ${ }^{3}$ and Takahisa Furukawa ${ }^{1,2}$ \\ ${ }^{1}$ Department of Developmental Biology, Osaka Bioscience Institute, ${ }^{2}$ Japan Science and Technology Agency, Core Research for Evolutional Science and \\ Technology, Osaka 565-0874, Japan, ${ }^{3}$ Department of Ophthalmology, Tokyo University School of Medicine, Tokyo 113-0033, Japan, and ${ }^{4}$ Laboratory for \\ Vertebrate Body Plan, Center for Developmental Biology, RIKEN Kobe, Kobe 650-0047, Japan
}

The molecular mechanisms underlying cell fate determination from common progenitors in the vertebrate CNS remain elusive. We previously reported that the OTX2 homeoprotein regulates retinal photoreceptor cell fate determination. While 0 tx 2 transactivation is a pivotal process for photoreceptor cell fate determination, its transactivation mechanism in the retina is unknown. Here, we identified an evolutionarily conserved $0 t x 2$ enhancer of $\sim 500 \mathrm{bp}$, named embryonic enhancer locus for photoreceptor Otx2 transcription (EELPOT), which can recapitulate initial Otx2 expression in the embryonic mouse retina. We found that the RAX homeoprotein interacts with EELPOT to transactivate $0 t x 2$, mainly in the final cell cycle of retinal progenitors. Conditional inactivation of Rax results in downregulation of Otx2 expression in vivo. We also showed that NOTCH-HES signaling negatively regulates EELPOT to suppress Otx2 expression. These results suggest that the integrated activity of cell-intrinsic and -extrinsic factors on EELPOT underlies the molecular basis of photoreceptor cell fate determination in the embryonic retina.

\section{Introduction}

Postmitotic neurons and glial cells are produced from a pool of cycling progenitors in an orderly fashion during development. Studies of cell fate determination in the vertebrate retina have uncovered several fundamental principles by which this is achieved (Livesey and Cepko, 2001). Retinal progenitor cells (RPCs) are multipotent subsets of progenitors and are therefore not limited to the generation of only one or two cell types (Turner and Cepko, 1987; Holt et al., 1988; Wetts and Fraser, 1988; Turner et al., 1990). In the developing brain, many progenitor cells were found to be able to give rise to several different cell types, including different types of neurons and glia, as seen in the developing retina (Walsh and Cepko, 1988, 1993). During verte-

Received June 16, 2011; revised Sept. 15, 2011; accepted Sept. 27, 2011.

Author contributions: T.F. designed research; T.F., Y.M., K.T., T.I., K.K., and T.T. performed research; T.F., R.S., D.K., S.A., and Y.T. contributed unpublished reagents/analytic tools; T.F., Y.M., K.T., T.I., and K.K. analyzed data; T.F., Y.M., and K.T. wrote the paper.

This work was supported by Core Research for Evolutional Science and Technology from Japan Science and Technology Agency, Grant-in-Aid for Scientific Research (B), and Young Scientists (B) from Ministry of Education, Culture, Sports, Science and Technology, The Takeda Science Foundation, The Uehara Memorial Foundation, Naito Foundation, Kato Memorial Bioscience Foundation, and Japan National Society for the Prevention of Blindness. We thank Akiko Tani, Mikiko Kadowaki, Aiko Ishimaru, Hiroko Abe, and Shawna Kennedy for technical assistance, and Dr. Miyazaki for providing us the CAG-CAT-Z mouse.

The authors declare no competing financial interests.

*Y.M. and K.T. contributed equally to this work.

Correspondence should be addressed to Takahisa Furukawa, Department of Developmental Biology, 0saka Bioscience Institute, 6-2-4 Furuedai, Suita, 0saka 565-0874, Japan. E-mail: furukawa@obi.or.jp.

K. Terada's present address: Department of Cellular Pharmacology, Graduate School of Medicine, Hokkaido University, Hokkaido 060-8638, Japan.

DOI:10.1523/JNEUROSCI.3109-11.2011

Copyright $\odot 2011$ the authors $\quad 0270-6474 / 11 / 3116792-16 \$ 15.00 / 0$ brate retinal development, retinal ganglion cells, horizontal cells, rod and cone photoreceptor cells, amacrine cells, bipolar cells, and Müller glial cells arise from multipotent RPC in a conserved order during development.

We previously reported that OTX2 homeoprotein controls photoreceptor cell fate. Otx 2 conditional knock-out (CKO) mice, whose $0 t x 2$ gene is inactivated in developing photoreceptors, showed a total loss of retinal photoreceptors and a pronounced increase of amacrine-like cells. However, retroviral gene transfer of Otx2 steered RPCs toward becoming photoreceptors, suggesting that OTX2 plays an instructive role in photoreceptor cell fate determination (Nishida et al., 2003). At early embryonic stages in vertebrates, Otx2 has widespread expression in the epiblast, but later in development, Otx2 expression becomes concentrated in the forebrain and midbrain neuroepithelium including the eye domain. Afterward, however, Otx2 expression is downregulated in the developing optic vesicle (Furukawa et al., 1997). When cone photoreceptor precursors begin to differentiate around embryonic day 11.5 (E11.5), Otx2 transcription is activated in cone photoreceptor precursors and exerts a critical role in photoreceptor cell fate determination (Nishida et al., 2003). Therefore, we think that understanding the transcriptional regulatory mechanisms of Otx2 in photoreceptor precursors will lead to the elucidation of a key molecular mechanism underlying photoreceptor cell fate determination.

In the current study, we investigated the transactivation mechanism of $0 t x 2$ in retinal photoreceptor precursors. We first found that OTX2 expression begins mainly in the final cell cycle of RPCs. We analyzed the regulatory region of the Otx2 gene 
during embryonic stages when Otx2 transcripts are distinctly expressed in the presumptive photoreceptor layer in contrast to postnatal stages when Otx2 expression shifts to the bipolar cell layer (Nishida et al., 2003; Koike et al., 2007). We then identified an $\sim 500$ bp cis-regulatory region we called embryonic enhancer locus for photoreceptor Otx2 transcription (EELPOT) that can recapitulate initial Otx2 transcription in early developing photoreceptors. We also provide evidence that the enhancer region is transactivated by RAX, which is a homeoprotein transcription factor expressed in RPCs, and negatively regulated by the HES family of molecules, which are bHLH transcription repressors. Our results suggest that the integrated functions of RAX homeoprotein and canonical NOTCH-HES signaling on a key enhancer is the foundation of embryonic photoreceptor cell fate determination.

\section{Materials and Methods}

\section{S-phase labeling}

For single S-phase labeling, timed pregnant female mice (E15.5) were injected intraperitoneally with 5-bromo-2'-deoxyuridine (BrdU) (50 $\mu \mathrm{g} / \mathrm{g}$ body weight) 1,2 , or $4 \mathrm{~h}$ before killing. For double S-phase labeling, timed pregnant female mice (E14.5) were injected intraperitoneally with 5-iodo-2'-deoxyuridine (IdU) (50 $\mu \mathrm{g} / \mathrm{g}$ body weight) followed by BrdU injection $(50 \mu \mathrm{g} / \mathrm{g}$ body weight) at $1.5,5.5,10$, and $14.5 \mathrm{~h}$ after IdU injection. Embryos were harvested $15 \mathrm{~h}$ after IdU injection. For BrdU and IdU immunostaining, sections were pretreated with $2 \mathrm{~N} \mathrm{HCl}$ for 15 min at $37^{\circ} \mathrm{C}$ before blocking. To detect IdU- and BrdU-positive cells, an antiBrdU antibody that recognizes both IdU and BrdU was used (BD Biosciences; 347580 ). An antibody that recognizes only BrdU was used to detect BrdU-incorporating cells (Santa Cruz; sc-56258). Combined use of the anti-BrdU antibody and the anti-BrdU/IdU antibody allowed us to identify only IdU-incorporating cells that had exited the S phase of cell cycle before the first BrdU injection.

\section{Cell culture, transfection, and luciferase assay}

Transient transfection was performed using LTX (Invitrogen) according to manufacturer's instructions. NIH3T3 cells were transfected with expression vectors encoding proteins of interest, a luciferase reporter plasmid connected to an EELPOT fragment, and an expression vector encoding $\beta$-galactosidase ( $\beta$-gal) as an internal control. Luciferase activity was measured using the Luciferase Assay System (Promega) according to manufacturer's instructions. Firefly luciferase activities were determined by three independent transfections and normalized by $\beta$-gal activity as an internal control.

\section{Immunostaining, in situ hybridization, and X-gal staining}

Mouse embryos and eye cups were fixed in $4 \%$ paraformaldehyde in PBS for either $30 \mathrm{~min}$ to $3 \mathrm{~h}$ (for immunostaining) or overnight (for in situ hybridization). The samples were cryoprotected, embedded, frozen, and sectioned (16-20 $\mu \mathrm{m}$ thick). Slides were incubated with blocking solution (4\% normal donkey serum and $0.5 \%$ Triton X-100 in PBS) for $1 \mathrm{~h}$, and then with primary antibodies for $4 \mathrm{~h}$ at room temperature. Slides were washed with PBS three times for $10 \mathrm{~min}$ each time and incubated with secondary antibodies for $2 \mathrm{~h}$ at room temperature. Rhodaminelabeled peanut agglutinin (PNA) (Vector Laboratories; RL-1072) was used for staining cone photoreceptors. 4',6-Diamino-2-phenylindole (DAPI) was used for staining DNA. TSAPLUS Fluorescence Kit (PerkinElmer; catalog \#NEL753001KT) was used for immunostaining of HES1 and HES5. Sections were boiled in $10 \mathrm{~mm}$ sodium citrate buffer, $\mathrm{pH}$ 6.0 , for $10 \mathrm{~min}$ for antigen retrieval and rinsed twice using $0.1 \mathrm{M}$ PBS, and immunostaining was performed according to manufacturer's instructions. Image acquisition and analysis of the signals obtained were performed using a Zeiss confocal LSM700 microscope. In situ hybridization and X-gal staining were performed essentially as described previously (Furukawa et al., 2002; Sanuki et al., 2010).

\section{Antibodies}

For immunostaining, anti- $\beta$-GAL antibody (Promega, Z3781, mouse monoclonal, 1:1000; Millipore Bioscience Research Reagents, AB1211, mouse monoclonal, 1:1000), anti-RHODOPSIN (LSL; LB-5597; rabbit polyclonal; 1:10,000), anti-OTX2 antibody (R\&D Systems; AF1979; goat polyclonal; 1:1000), anti-HES1 antibody (Santa Cruz; sc-25393; rabbit polyclonal; 1:100), anti-HES5 antibody (Santa Cruz; sc-25395; rabbit polyclonal; 1:100), anti-PAX6 antibody (Developmental Studies Hybridoma Bank; P3U1; mouse monoclonal; 1:200), anti-FLAG antibody (M2) (Sigma-Aldrich; F3165; mouse monoclonal; 1:1000), and anti-BrdU antibodies (BD Biosciences, 347580, mouse monoclonal, 1:100; Santa Cruz, sc-56258, rat monoclonal, 1:100) were used as primary antibodies. Anti-RAX antibody was raised against a peptide (PKAPAGGSESSPPAAPGFVPEYEATRP) followed by affinity purification using the same peptide (guinea pig polyclonal; 1:1000). Anti-CRX antibody was raised against a fusion protein expressed in Escherichia coli using a construct in which the C-terminal portion of mouse CRX (residues 105-299) was subcloned into pET28a, followed by affinity purification (rabbit polyclonal; 1:4000). Cy3-conjugated anti-rabbit IgG (Jackson ImmunoResearch; 711-165-152), Cy3-conjugated anti-mouse IgG (Jackson ImmunoResearch; 711-165-150), Alexa Fluor 647-conjugated anti-goat IgG (Invitrogen; A21447), FITC-conjugated anti-goat IgG (Jackson ImmunoResearch; 705-095-147), FITC-conjugated anti-rabbit IgG (Jackson ImmunoResearch; 712-095-153), and Cy3-conjugated anti-guinea pig IgG (Millipore; AP193C) were used as secondary antibodies.

\section{Chromatin immunoprecipitation assay}

Chromatin immunoprecipitation (ChIP) assay was performed essentially as described previously (Shang et al., 2000). Mouse embryonic retinas were cross-linked with $1 \%$ formaldehyde in PBS at room temperature for $15 \mathrm{~min}$. Retinas were rinsed with ice-cold PBS twice and incubated with $0.125 \mathrm{M}$ glycine in PBS for $10 \mathrm{~min}$. The immunoprecipitated chromatin DNA was analyzed by semiquantitative PCR or real-time PCR. Primer sequences are as follows: for $H s p 3$ region, forward (F), 5'-CTCAAACCACATGCAAGGGTATC- ${ }^{\prime}$, and reverse (R), 5' ${ }^{\prime}$-TAAG GGAAGAAGAAGTAGAGGTC-3'; EELPOT, F, 5' -TGATGCCCAAAA GGTATCCAAA-3', and R, 5' -TAAAATCACCCCTAAACACAGG-3'; Otx2 gene, F, 5' -CTAGGGCACAGCTCGACGTTCTG-3' , and R, 5' -CC TGGTTCATATGATTAAGGAGG-3'. Twenty-eight to 30 cycles of amplification were used for PCR. Anti-RAX antibody $(6 \mu \mathrm{g})$ and anti-HES1 $(6 \mu \mathrm{g})$ antibody were also used for ChIP assay.

\section{Real-time PCR}

Total RNA was reverse-transcribed by SuperScript II (Invitrogen) using random primers. Real-time PCR was performed using the Thermal Cycler Dice Real-Time System (Takara) and SYBR GreenER qPCR SuperMix Universal (Invitrogen), according to the manufacturer's protocols. Quantified RNA values were normalized with those of $\beta$-actin RNA. Quantitative analysis was performed at least three times and shown with SEs. Primer sequences are as follows: for Otx2, F, 5' -GGTATGGACTTGCTGCATCC-3', and R, 5' -CG AGCTGTGCCCTAGTAAATG-3'; Hes1, F, 5' -TGCCAGCTGATATAAT GGAGAA-3', and R, 5' -CCATGATAGGCTTTGATGACTTT-3'; LacZ, F, 5'-ATTGAACTGCCTGAACTACCG-3' , and R, 5' -TGAGGTTTTCCGCC AGAC- $3^{\prime}$; $\beta$-actin, F, 5'-CGTGCGTGACATCAAAGAGAA- $3^{\prime}$, and R, 5'-TGGATGCCACAGGATTCCAT-3'.

\section{Retina explant culture}

Embryonic mouse eyes, including the retina and lens, were transferred to nucleopore polycarbonate membrane filters, $0.2 \mu \mathrm{m}$ pore size (Whatman; 110606) after the retinal pigment epithelium (RPE) was removed, and cultured for $6 \mathrm{~d}$ in DMEM supplemented with $10 \% \mathrm{FBS}, 100 \mathrm{IU} / \mathrm{ml}$ penicillin, and $100 \mu \mathrm{g} / \mathrm{ml}$ streptomycin. Retinas were fixed in $4 \%$ paraformaldehyde in PBS for $3 \mathrm{~h}$. The samples were cryoprotected, embedded, and used for immunostaining.

\section{Plasmids}

pcDNA3-3FLAG-Rax, pcDNA3-3FLAG-Otx2, pcDNA3-3FLAG-Crx, pcDNA3-3FLAG-NeuroD1, pcDNA3-3FLAG-Mash1, pcDNA3-3FLAGMath5, pcDNA3-Math3, pCS2(+)-Pax6-4HA, pCS2(+)-Chx10-4HA, pCS2(+)-Ngn2, pCS2(+)-Hes1-4HA, pCS2(+)-Hey1, and pCS2(+)Hes 5 were used. A total of $0.5 \mu \mathrm{g}$ of each plasmid was used for transfection. A total of $0.1 \mu \mathrm{g}$ of $p C S 2(+)-L a c Z$ or EELPOT-luc was used. $p C S 2(+)$ and $p c D N A 3$ are CMV promoter-directed expression vectors. 


\section{Animal care}

All procedures conformed to the Association for Research in Vision and Ophthalmology Statement for the Use of Animals in Ophthalmic and Vision Research, and were approved by the Institutional Safety Committee on Recombinant DNA Experiments and the Animal Research Committee of Osaka Bioscience Institute. Mice were housed in a temperature-controlled room at $22^{\circ} \mathrm{C}$ with a $12 \mathrm{~h}$ light/dark cycle. Fresh water and rodent diet were available at all times.

\section{Mouse lines and transgenic or targeting constructs}

Rax flox mouse line. To generate the Rax flox mouse line, we obtained genomic DNA clones covering the Rax gene locus by a screen of the $129 / S v E v$ mouse genomic DNA library (Stratagene). We subcloned a $\sim 13 \mathrm{~kb}$ Rax genomic fragment, inserted a flox cassette upstream of exon 1 and intron 2 , cloned it into a modified $P P N T$ vector to make a targeting construct, and transfected the linearized targeting construct into the TC1 embryonic stem (ES) cell line (Deng et al., 1996). The culture, electroporation, and selection of TC1 were performed as previously described (Sato et al., 2008). ES cells that were heterozygous for the targeting gene disruption were microinjected into C57BL/6 blastocysts to obtain chimeric mice.

Otx2 bacterial artificial chromosome \#2 transgenic mouse line. To generate an Otx2 bacterial artificial chromosome (BAC) \#2 transgenic mouse, a mouse Otx2 genomic BAC clone (RP23-153H20) was used to generate a BAC reporter transgene in which a $L a c Z$ gene with the $S V 40$ $\operatorname{poly}(A)$ signal $(p A)$ was inserted before the translation start site of the Otx2 gene.

Crx-CreERT2 transgenic mouse line. $\beta$-gal in the $p \beta$-gal-Basic vector (Clontech) was replaced with the CreERT2 fragment, which was excised from $p C r e-E R T 2$ using EcoRI (Feil et al., 1997). The $\sim 2 \mathrm{~kb}$ promoter fragment of the Crx gene (Furukawa et al., 2002) was inserted before CreERT2 to generate a Crx-CreERT2 transgene.

EELPOT-Otx2 and EELPOT-LacZ transgenic mouse lines. EELPOT located at $\sim 9 \mathrm{~kb}$ upstream of the $O t x 2$ gene was connected to a heat shock promoter 68 (Hsp68)-Otx2-pA cassette or a Hsp68-LacZ-pA cassette (Kothary et al., 1989). The resulting constructs were used for generating transgenic mice lines.

Hes1 transgenic mouse. A FLAG-tag encoding sequence was introduced in front of the initiation codon of rat Hes1 cDNA to generate FLAG-Hes1, and this FLAG-Hes1 fragment was connected after the $\sim 2$ $\mathrm{kb}$ promoter fragment of the Crx gene. The resulting construct was used for generating transient transgenic mice, which were harvested at E13.5.

The purified constructs for generating transgenic mice were injected into the pronuclei of $\mathrm{C} 57 \mathrm{BL} / 6 \times \mathrm{C}_{3} \mathrm{H} \mathrm{F}_{1}$ hybrid eggs at the one-cell stage followed by implantation into pseudopregnant foster mothers (ICR; Japan SLC).

The Otx2 flox mouse line. CAG-CAT-Z mouse line and BAC \#1 reporter transgenic mice were described previously (Sakai and Miyazaki, 1997; Tian et al., 2002; Kurokawa et al., 2004a).

The EELPOT (CR1) region in the BAC \#2 clone was replaced with an EGFP-poly $(A)$ signal $(p A)$ cassette using the $p L D 53 . S C A-E-B$ shuttle vector by homologous recombination (Yang et al., 1997; Gong et al., 2002; Sato et al., 2007), creating BAC $\# 2 \Delta C R 1$. Subsequently, the Hsp1-3 region in the resulting $\mathrm{BAC}$ was replaced with a Cre-pA cassette using the pLD53.SCA-Cre- $B$ shuttle vector by homologous recombination, creating a $B A C \# 2 \Delta C R 1, \Delta H s p 1-3$. The Hsp1-3 region in BAC \#2 was replaced with an EGFP-pA cassette using the $p L D 53 . S C A-E-B$ shuttle vector by homologous recombination, creating a BAC $\# 2 \Delta$ Hsp 1-3.

Hspl was obtained as an EcoRV/EcoRV $12 \mathrm{~kb}$ DNA fragment (nucleotides $170,477-182,321$ in the Otx2 locus). Hsp3 was obtained as a BamHI/BamHI $7 \mathrm{~kb}$ DNA fragment (nucleotides 158,220-165,764). Hsp1-1 was obtained as an EcoRV/HincII $5 \mathrm{~kb}$ DNA fragment (nucleotides 170,477-175,530), and Hsp1-3 was obtained as a HincII/EcoRV 5 kb DNA fragment (nucleotides 177,767-182,321). Hsp2, Hsp4, Hsp1-2, and CR2 were produced by PCR using primers as follows: for $H s p 2, \mathrm{~F}$, 5' -CGCTCGAGTTACCGTTCTGGAGAGCACCAC-3' , andR, 5' -ATGA ATTCGTTATGTGTTGGCTGTGGGTAT- $3^{\prime}$; for $H s p 4$, F, $5^{\prime}$-ACTCGA GGATATCAGCCATAATGTGGGAAT- ${ }^{\prime}$, andR, 5' $^{\prime}$-AAGTCGACGATT CGCCTGGAGTGGACAGGGTCAG-3'; for Hsp1-2, F, 5' -CAGCTACC
GGTGAGGACACGACTCA-3', and R, 5' -ATCCCGGGATTCAGCATT GTCACCAGATTT-3'; and for CR2, F, 5' -AAGTCGACGAAAAT CCCC GCCAGACCGAAGCC-3', and R, 5'-AAGGATCCAACTCATCGTAT TTGCTTTTACAA-3'.

\section{Statistical analysis}

Data are presented as means \pm SD. Statistical significance was calculated with a Student's $t$ test.

\section{Results}

\section{OTX2 expression begins in the final cell cycle of RPCs}

Cell fate determination in the vertebrate CNS is thought to occur largely during the final cell cycle, suggested from the transplantation study using the developing ferret neocortex (McConnell and Kaznowski, 1991). In the developing retina, lineage studies showed that the RPCs remain uncommitted to a specific cell fate until the final cell division (Turner and Cepko, 1987; Turner et al., 1990). To clarify the timing of the onset of OTX2 expression in the cell cycle of RPCs, we examined in which cell cycle phase Otx2 transcription begins in the embryonic mouse retina by a combination of immunohistochemistry and double S-phase labeling (Fig. 1). We injected BrdU into E15.5 pregnant mice to label cells in $\mathrm{S}$ phase (Fig. $1 A$ ). Embryos were harvested $1 \mathrm{~h}$ (Fig. $1 A, B, E$ ), $2 \mathrm{~h}$ (Fig. $1 A, C, F$ ), or $4 \mathrm{~h}$ (Fig. $1 A, D, G)$ after BrdU injection. Previous studies reported that BrdU-labeled retinal cells 1, 2, or $4 \mathrm{~h}$ after BrdU injection are in the $S, G_{2}$, or $M$ phase, respectively (Alexiades and Cepko, 1996; Dyer et al., 2003). We found that OTX2 is expressed in BrdU-positive cells (Fig. $1 B-D, H$ ), suggesting that OTX2 expression begins before cell cycle exit. We also examined CRX expression, which is an OTX2 downstream gene expressed in photoreceptor precursors, and observed few or no CRXpositive cells in the BrdU-positive cells, suggesting that CRX expression begins after cell cycle exit (Fig. $1 E-H)$. We observed that the number of OTX2 and BrdU double-positive cells increased in proportion to the amount of time after BrdU injection (Fig. $1 \mathrm{H}$ ). These observations fit well with the results previously reported that OTX2 expression begins in the late $S$ to early $\mathrm{G}_{2}$ phase of cell cycle and CRX expression begins in the postmitotic phase (Trimarchi et al., 2008).

We further extended our analysis to estimate the timing of OTX2 expression by double labeling. We injected IdU into E14.5 pregnant mice followed by BrdU injection 1.5, 5.5, 10, and $14.5 \mathrm{~h}$ after IdU injection to label progenitor cells in S phase (Fig. $1 \mathrm{~A}$ ). The IdU-positive and BrdU-negative cells are the cells that exit their $\mathrm{S}$ phase before the first BrdU injection at $1.5 \mathrm{~h}$ after IdU injection. We chased the fate of these IdU-positive and BrdUnegative cells until $15 \mathrm{~h}$ later when they are supposed to enter the $\mathrm{G}_{1}$ or $\mathrm{G}_{0}$ phase (Alexiades and Cepko, 1996; Dyer et al., 2003). We examined whether IdU-positive, BrdU-negative, and OTX2positive cells became postmitotic by coimmunostaining using an antibody against CRX. We observed at least $\sim 70 \%$ of IdUpositive, BrdU-negative, and OTX2-positive cells were also CRX positive (Fig. 1I,J). These results strongly suggest that OTX2 expression begins mainly during the last cell cycle just before they exit the cell cycle.

Our observations suggest that molecular mechanisms governing photoreceptor cell fate determination during the final $\mathrm{RPC}$ cell division and molecular mechanisms regulating Otx2 expression are closely related. We then investigated the regulatory mechanism of $\mathrm{Otx} 2$ expression in the developing retina. 
A Time
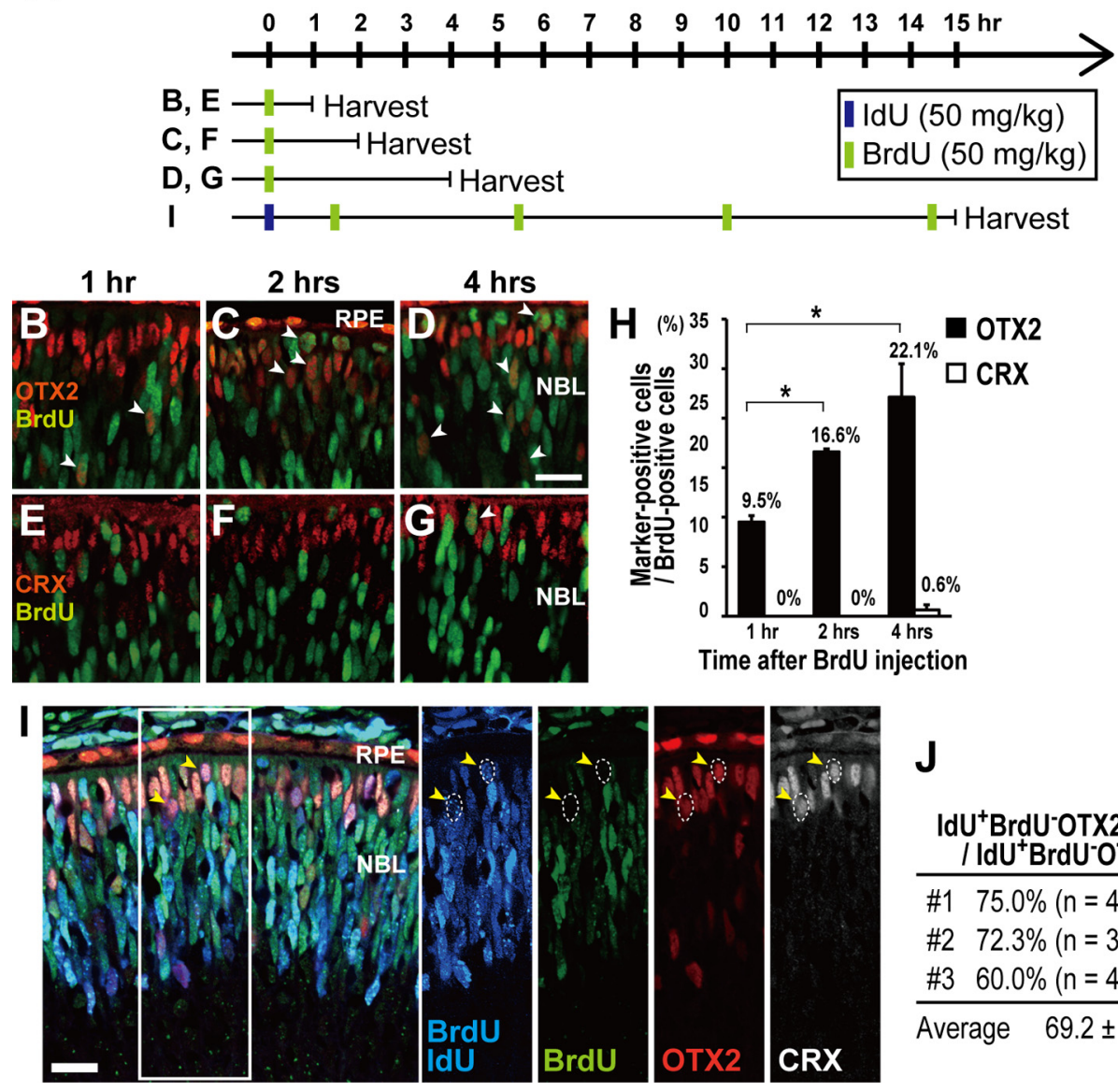

J

Figure 1. OTX2 expression begins during the final RPC cell cycle. A, Schematic diagram of single or double S-phase labeling protocols used to administer thymidine analogs (IdU and/or BrdU) to label cells. Timed pregnant female mice atE14.5 were subjected to BrdU injection and at E15.5 were subjected to IdU and BrdU injection. $\boldsymbol{B}-\boldsymbol{G}$, Immunostaining of retinal section of embryos that were injected with BrdU $1 \mathrm{~h}(\boldsymbol{B}, \boldsymbol{E}), 2 \mathrm{~h}(\boldsymbol{C}, \boldsymbol{F})$, or $4 \mathrm{~h}(\boldsymbol{D}, \boldsymbol{G})$ before harvest. Retinal sections were stained with an anti-BrdU antibody and an anti-0TX2 antibody $(\boldsymbol{B}-\boldsymbol{D})$, or an anti-BrdU antibody and an anti-CRX antibody $(\boldsymbol{E}-\boldsymbol{G})$. The white arrowheads indicate BrdU-positive and 0TX2-positive cells $(\boldsymbol{B}-\boldsymbol{D})$ or BrdU-positive and CRX-positive cells ( $\boldsymbol{G}$ ). RPE, Retinal pigment epithelium; NBL, neuroblastic layer. $\boldsymbol{H}$, Ratio of the number of BrdU- and OTX2-positive cells $\left(0 \mathrm{TX} 2^{+} / \mathrm{BrdU}^{+}\right)$, or BrdU- and CRX-positive cells $\left(\mathrm{CRX}^{+} / \mathrm{BrdU}^{+}\right)$to the total number of BrdU-positive cells, counted at each time point. Data are means \pm SEM. $n=1505$ cells from 9 sections $\left(1 \mathrm{~h}, 0 \mathrm{TX} 2^{+} / \mathrm{BrdU}{ }^{+}\right), n=524$ cells from 3 sections $\left(2 \mathrm{~h}, 0 \mathrm{TX2}{ }^{+} / \mathrm{BrdU}^{+}\right), n=519$ cells from 4 sections $\left(4 \mathrm{~h}, 0 \mathrm{TX2}{ }^{+} / \mathrm{BrdU}{ }^{+}\right), n=$ 1240 cells from 6 sections $\left(1 \mathrm{~h}, \mathrm{CRX}^{+} / \mathrm{BrdU}{ }^{+}\right), n=1417$ cells from 6 sections $\left(2 \mathrm{~h}, \mathrm{CRX}^{+} / \mathrm{BrdU}{ }^{+}\right)$, and $n=765$ cells from 8 sections $\left(4 \mathrm{~h}, \mathrm{CRX}^{+} / \mathrm{BrdU}^{+}\right)$. ${ }^{*} p<0.001$, Student's $t$ test. II, IdU- and BrdU-double-labeled retinas were immunostained with an anti-BrdU antibody, which stains both BrdU and IdU (blue, IdU-and/or BrdU-labeled cells); an anti-BrdU antibody, which stains only BrdU (green); an anti-0TX2 antibody (red); and an anti-CRX antibody (white). IdU was injected once $15 \mathrm{~h}$ before harvest while BrdU was injected every 4 or $5 \mathrm{~h}$ to label cells in $\mathrm{Sphase}$ as represented in (A). The yellow arrowheads indicate IdU-positive, BrdU-negative, OTX2-positive, and CRX-positive cells delineated by the dotted circle. J, Percentage of IdU-positive, BrdU-negative, 0TX2-positive, CRX-positive cells (IdU ${ }^{+} / \mathrm{BrdU}^{-} / \mathrm{OTX2}^{+} / \mathrm{CRX}^{+}$) in the IdU-positive, BrdU-negative, OTX2-positive (IdU ${ }^{+} / \mathrm{BrdU}^{-} / \mathrm{OTX2}^{+}$) cell population. Most IdU ${ }^{+} / \mathrm{BrdU}^{-} / \mathrm{OTX2}^{+}$cells were CRX-positive, which is a postmitotic photoreceptor precursor marker. $n=126$ cells on 11 sections of 4 retinas from 3 animals (\#1, \#2, and \#3) were analyzed. Data are means \pm SEM. Scale bars, $20 \mu \mathrm{m}$.

\section{Identification of $O t x 2$ enhancer in embryonic photoreceptor precursors}

To identify the mouse Otx2 regulatory locus directing expression in the embryonic photoreceptor precursors, we first analyzed two transgenic mouse lines harboring BACs, containing the mouse Otx2 gene inserted with a $\beta$-galactosidase ( $L a c Z$ ) reporter (Fig. $2 A)$. The BAC \#1 line was previously established to investigate Otx2 cis-regulatory elements important for developing forebrain and midbrain expression (Kurokawa et al., 2004a,b). The BAC \#2 clone, spanning $-150 \mathrm{~kb} 5^{\prime}$-upstream to $+70 \mathrm{~kb} 3^{\prime}$-downstream with respect to the site corresponding to the initiation codon of the $O t x 2$ gene, was inserted with $L a c Z$ immediately before the Otx2 initiation codon site. At E13.5, endogenous Otx2 transcripts were observed in the outer region of the developing mouse retina, a presumptive photoreceptor layer, by in situ hybridization (Fig. $2 B$ ). Similarly, we observed LacZ-positive cells in the presumptive photoreceptor layer in both the BAC \#2 germline-transmitted transgenic line retina and $\mathrm{BAC} \# 2$ transient transgenic retina at E13.5 (data not shown; Fig. 2C). We observed LacZ-positive cells in the presumptive photoreceptor layer during postnatal stages in the retinas of $\mathrm{BAC} \# 2$ transgenic mice, indicating that $\mathrm{BAC} \# 2$ recapitulates Otx2 expression in the developing retina (Fig. $2 D, E$ ). In contrast, we did not observe a significant number of LacZ-positive cells in the E13.5 retina of BAC \#1 harboring BAC spanning $-170 \mathrm{~kb} \mathrm{5'-}$ upstream to $-30 \mathrm{~kb} 5^{\prime}$-upstream of the Otx2 gene (Fig. 2 F), suggesting that the $O t \times 2$ regulatory region resides between -30 and $+70 \mathrm{~kb}$ of the Otx2 gene.

To further narrow down the critical region for the Otx2 regulatory activity, we examined the $-32 \mathrm{~kb} 5^{\prime}$-upstream region of the Otx2 gene first. We prepared DNA fragments of up to $\sim 12 \mathrm{~kb}$ in length overlapping each other, spanning from $-32 \mathrm{~kb}$ to the OTX2 initiation codon site of the Otx2 gene (Fig. 2A). We fused each of these DNA fragments to the $L a c Z$ gene or the minimal mouse heat shock protein 68 promoter connected immediately before $L a c Z$, and named them as $H s p 1,2,3$, and 4, respectively (Fig. 2A). We generated transient transgenic mice injected with 
A

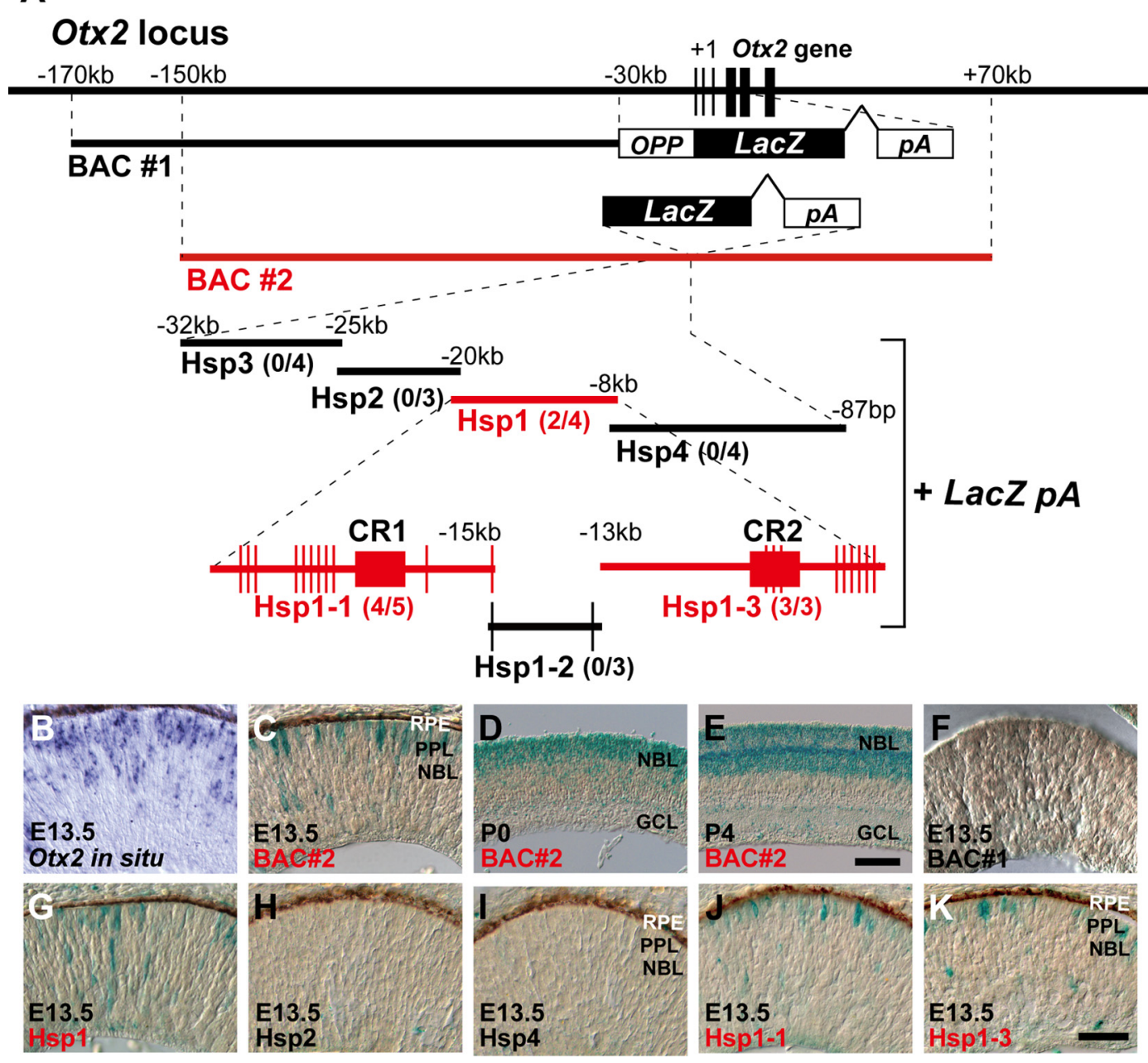

Figure 2. Regulatory region of Otx2 expression is located in the $5^{\prime}$-upstream region of $0 t \times 2$ gene. $A$, Schematic diagram of the mouse $0 t x 2$ locus and BAC reporter constructs, BAC\#1 and BAC\#2, containing a genomic region around the $0 t x 2$ locus. BAC $\# 2, H s p 1, H s p 1-1$, and $H s p 1-3$ represented by red color indicate that these regions recapitulate 0 tx2 expression in the embryonic mouse retina of transgenic mouse. The vertical bars on Hsp1-1, Hsp1-2, Hsp 1-3 represent potential OTX2-binding sites. CR1 and CR2 are evolutionarily conserved from opossum to human. A of the initiation codon (ATG) is taken as +1 . Number of mice exhibiting $\beta$-gal expression among transient transgenic mice positive for a transgene is indicated in parentheses. $B$, Expression pattern of 0 tx $2 \mathrm{mRNA}$ in the

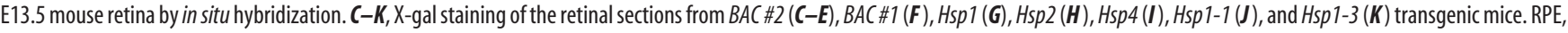
Retinal pigment epithelium; PPL, presumptive photoreceptor layer; NBL, neuroblastic layer. The vertical line is Otx2-binding site. Scale bars: $\boldsymbol{D}, \boldsymbol{E}, 100 \mu \mathrm{m} ; \boldsymbol{B}, \boldsymbol{C}, \boldsymbol{F}-\boldsymbol{K}, 50 \mu \mathrm{m}$.

these DNA constructs, and harvested embryos at E13.5. We observed LacZ-positive cells only in the retinas of $H s p 1$-injected mice in a similar pattern to Otx2 expression, suggesting that Hsp 1 contains a regulatory region responsible for Otx2 expression in the presumptive photoreceptor layer of the retina at E13.5 (Fig. $2 B, C, G-I)$. We divided Hspl into three fragments with short overlapping regions, and produced $L a c Z$ reporter constructs, designated Hsp1-1, Hsp1-2, and Hsp1-3 (Fig. 2A). We generated transient transgenic mice using these constructs, and detected LacZ-positive cells in the presumptive photoreceptor layer of the retina of mice injected with Hsp1-1 or Hsp1-3 (Fig. 2J,K). It should be noted that multiple potential OTX2-binding consensus sites are present in both Hsp1-1 and Hsp1-3 regions (Fig. 2A), suggesting that these OTX2-binding sites may serve as an OTX2 auto-positive feedback.

To confirm whether the Hsp1 region in BAC \#2 is responsible for Otx2 expression in the retina at E13.5, we prepared a BAC \#2 construct deleting the region corresponding to $H s p 1$, designated $\mathrm{BAC} \# 2 \Delta H s p 1$. We did not observe a significant number of LacZpositive cells in the retina of mice injected with the BAC \#2 $\Delta H s p 1$ transgene, suggesting that the Hsp1 region is necessary for Otx2 expression in the developing retina at E13.5 (Fig. $3 A, A^{\prime}$ ). We then searched for conserved regions among species in Hspl-1 or Hsp1-3. We found two $\sim 500$ bp evolutionarily well conserved regions from opossum to human (Fig. $3 E$ ), and called them conserved region 1 (CR1) and 2 (CR2), in Hsp1-1 and Hsp1-3, respectively (Fig. $2 A$ ). Since $C R 2$ contains at least three potential OTX2-binding sites, we focused more on CR1 than on CR2. To evaluate the importance of $C R 1$, we first prepared the BAC \#2 construct deleting both $C R 1$ and Hsp1-3 to examine whether these regions are required for $H s p 1$ to induce gene expression in the developing retina. We did not observe a significant number of LacZpositive cells in the retina of transgenic mice with CR1- and Hsp1-3deleted BAC \#2 (Fig. $3 B, B^{\prime}$ ). We then deleted CR1 or Hsp1-3 to further examine the significance of $C R 1$ or Hsp1-3 for induction of LacZ (Fig. 3C,D). While we observed LacZ-positive cells in the BAC $\# 2 \Delta$ Hsp1-3 retinas, we failed to observe LacZ-positive cells in BAC $\# 2 \Delta \mathrm{CR} 1$ transgenic mice (Fig. $3 C-D^{\prime}$ ). Although the Hsp1-3 transgene alone can produce a signal (Fig. $2 K$ ), we assume that BAC \#2 may contain a silencer region that suppresses Hsp 1-3 activity. These results suggest that $C R 1$ has a significant role in the regulatory activity of Otx2 in the developing retina at E13.5. 

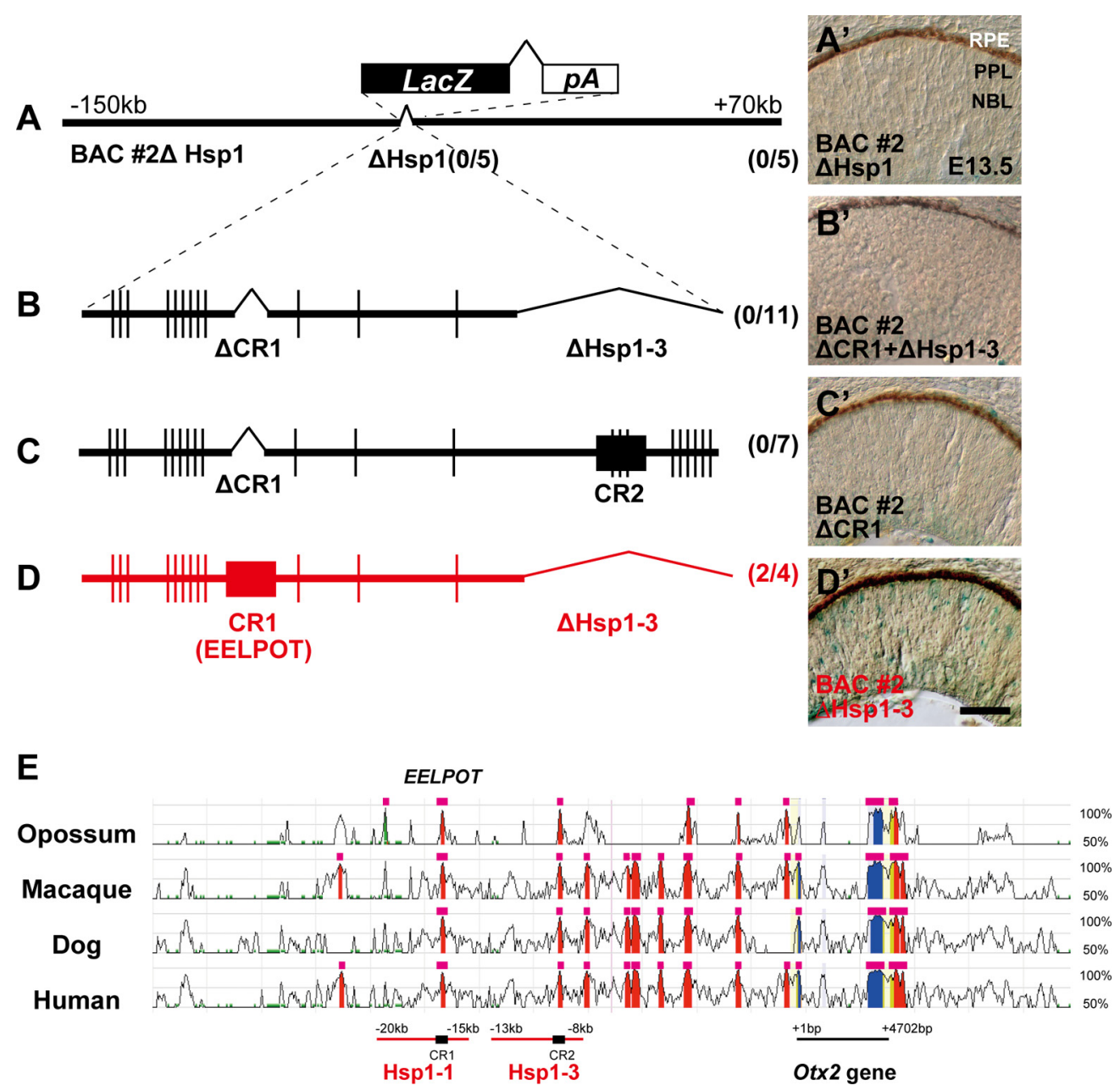

Figure 3. The CR1 region is necessary for Otx2 expression in the developing retina. $\boldsymbol{A}-\boldsymbol{D}^{\prime}$, Schematic diagram of BAC $\# 2$ construct with deletions of $H s p 1(\boldsymbol{A}), C R 1$ and $H s p 1-3$ (B), $C R 1$ (C), or Hsp1-3

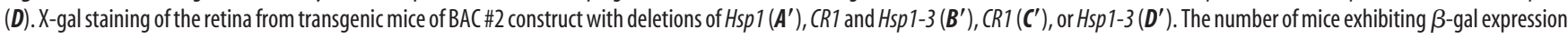
among transient transgenic mice positive for the transgene is indicated in parentheses. RPE, Retinal pigment epithelium; PPL, presumptive photoreceptor layer; NBL, neuroblastic layer. The vertical line represents 0 tx2-binding site. Scale bars, $50 \mu \mathrm{m}$. E, The -32 to $+12 \mathrm{~kb}$ region of the mouse Otx2 gene is compared with the opossum (Monodelphis domestica), macaque (Macaca mulatta), dog (Canis lupus familiaris), and human (Homo sapiens) syntenic regions (ECR browser; http://www.dcode.org/). Conservation rate between mouse and each species is plotted by percentages and appearing in the figure in only the genomic regions with $50-100 \%$ conservation. Those with $>95 \%$ sequence identity in contiguous 100 bp windows of genomic sequence (red areas, intergenic regions; blue, coding exons; yellow, UTRs) are highlighted by pink bars. Hsp1-1 and Hsp 1-3, which showed Otx2-like reporter expression in transgenic assays, are shown with evolutionarily conserved $C R 1$ and $C R 2$ regions. The $\sim 500$ bp CR1 (EELPOT) and CR2 regions are indicated by black boxes.

\section{An evolutionarily conserved genomic region is critical for Otx2 transactivation}

We then examined whether CR1 is sufficient to recapitulate Otx2 expression by a transient transgenic assay using the LacZ reporter in which a CR1 DNA fragment is linked with an Hsp68-LacZ-pA cassette to produce CR1-LacZ (Fig. 4A). We observed five CR1$L a c Z$ transgenic mice expressing $L a c Z$ in the outer region of the retina of 11 transient transgenic mice (Fig. $4 B, C$ ). Next, we generated three germline-transmitted CR1-LacZ transgenic mice lines to further investigate this region. Since all of these lines exhibited similar LacZ expression patterns (data not shown), we performed further analysis using the transgenic $C R 1$ line 1 , which exhibited slightly more LacZ-positive cells than the other CR1 lines. We observed LacZ-positive cells in the presumptive photoreceptor layer of the retina from $C R 1$ line 1 (Fig. $4 D, E$ ), although the number of LacZ-positive cells observed in the CR1 line 1 transgenic mouse seemed to be smaller compared with that in the BAC \#2 transgenic mouse. It should be noted that CR1-driven LacZ expression decreases significantly after late embryonic stages (Fig. $4 F, G$ ), suggesting that EELPOT plays a crucial role for the initial Otx2 expression but not for the maintenance of $O t \times 2$ expression. We also examined whether CR2 has a similar transcriptional activity to EELPOT by generating transient transgenic mice using a CR2-LacZ construct (Fig. 4H). We observed LacZpositive cells throughout the retina at E13.5, suggesting that the $C R 2$ region contains a regulatory region other than that for $O t \times 2$ (Fig. 4I). We observed LacZ-positive cells in the outer region of the CR2-LacZ transgenic retina at P0 (Fig. $4 J$ ) in contrast to the absence of LacZ-positive cells in the CR1-LacZ transgenic P0 retina (Fig. $4 G$ ). These results suggest that $C R 2$ has different transcriptional regulatory activities than CR1 in embryonic retinal development.

We further examined whether CR1 can recapitulate OTX2 expression in the developing retina by immunohistochemistry (Fig. $4 K-P)$. We measured the percentage of OTX2-positive cells in LacZpositive cells (Fig. 4Q). We observed that most LacZ-positive cells were OTX2-positive, indicating that $C R 1$ can at least partially recapitulate OTX2 expression in the embryonic retina (Fig. $4 N-Q$ ). Based on these observations, we named CR1 embryonic enhancer locus for photoreceptor Otx2 transcription (EELPOT). 

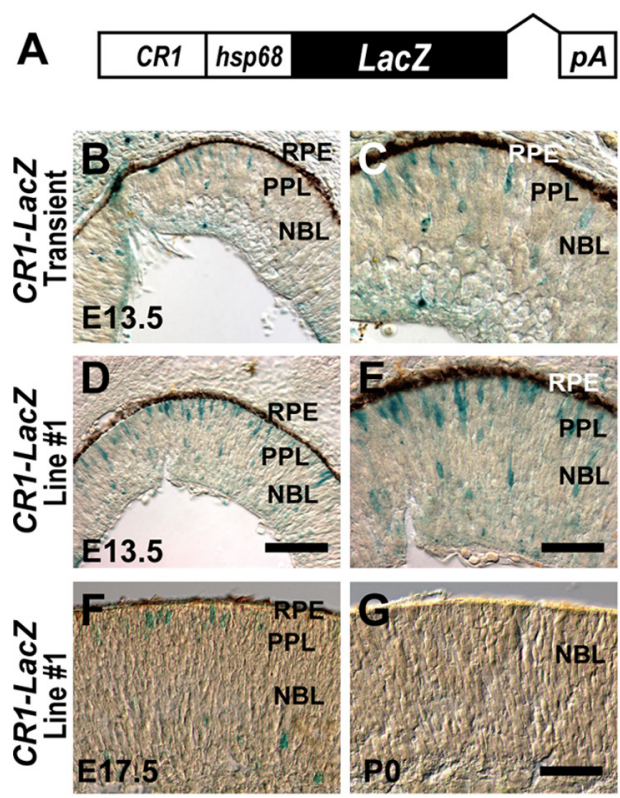

H
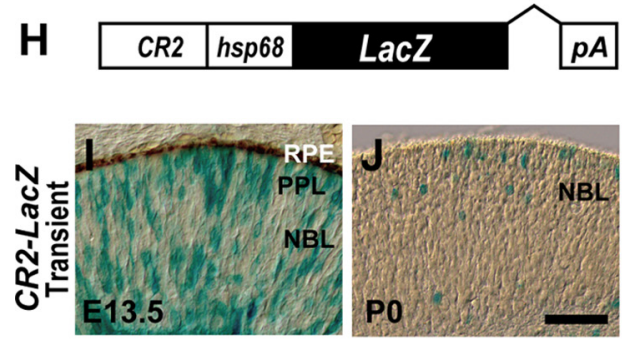
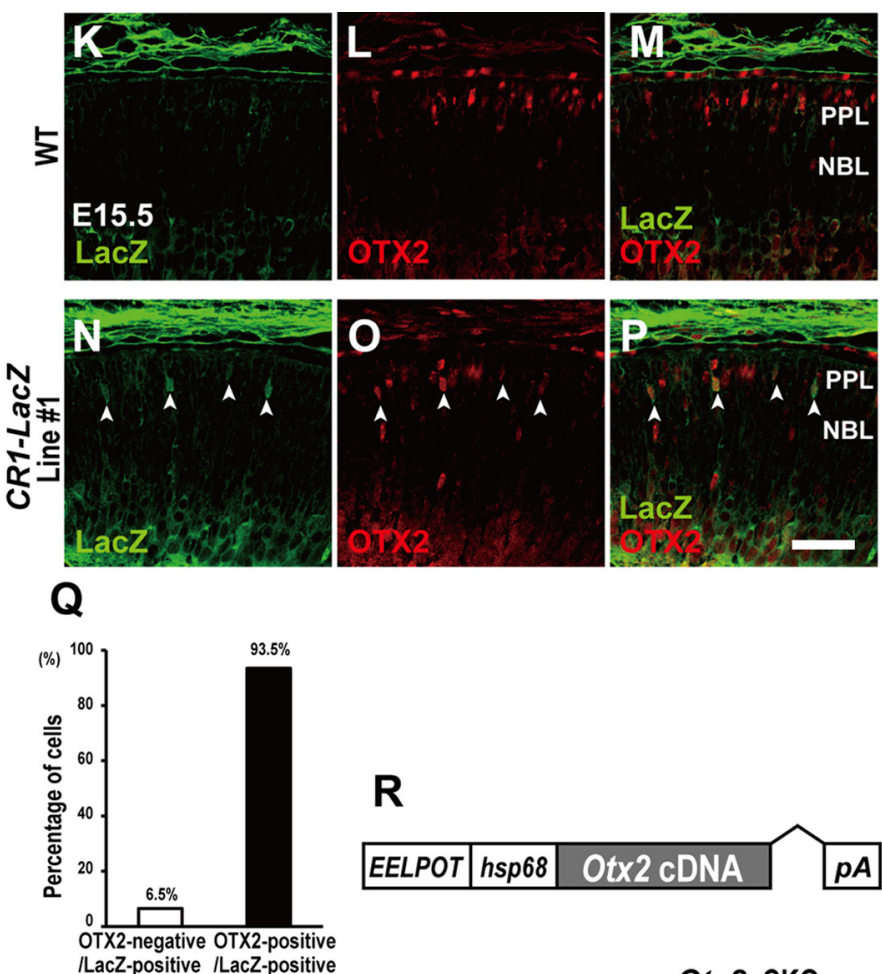

$\mathbf{R}$
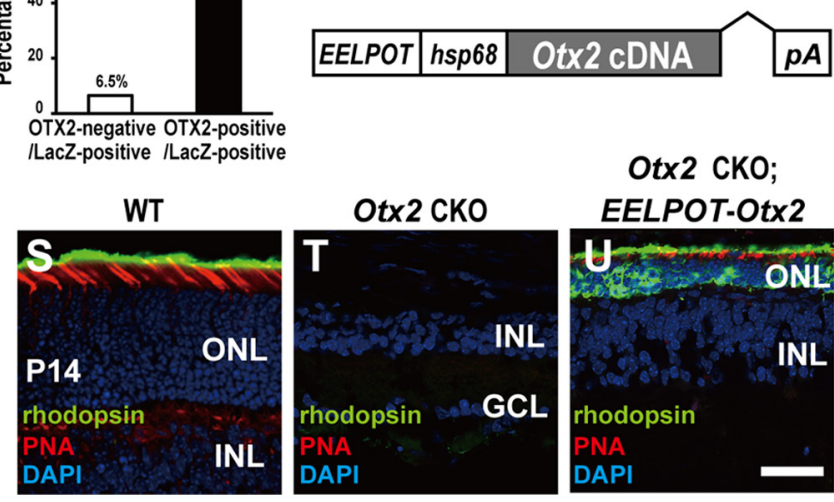

Otx2 CKO; EELPOT-Otx2

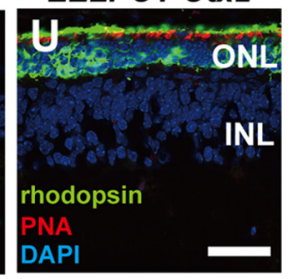

Figure 4. CR1 (EELPOT) recapitulates OTX2 expression in the embryonic mouse retina. $A$, Structure of CR1 (EELPOT)-LacZ reporter construct. $B$ - $G, X$-gal staining of retinal sections from transient transgenic CR1 (EELPOT)-LacZ mice (B, C) and germline-transmitted CR1 (EELPOT)-LacZ transgenic mouse line (line 1) (D, E) at E13.5. (C, E), Higher magnifications of (B) and (D). X-gal staining of the retinal sections from E17.5 (F) or PO (G) EELPOT-LacZ transgenic mouse line 1. RPE, Retinal pigment epithelium; PPL, presumptive photoreceptor layer; NBL, neuroblastic layer. Scale bars: $\boldsymbol{D}$, 100 $\mu \mathrm{m} ; \boldsymbol{E}, \mathbf{G}, 50 \mu \mathrm{m}$. $\boldsymbol{H}$, Structure of CR2-LacZ reporter construct. I, J, X-gal staining of retinal sections from transient transgenic CR2-LacZ mice of E13.5 (I) or P0 (J). Scale bar: J, $50 \mu \mathrm{m}$. $\boldsymbol{K}$ - $\boldsymbol{P}$, Immunostaining of retinal sections from WT $(\boldsymbol{K}-\boldsymbol{M})$ or line 1 mice $(\boldsymbol{N}-\boldsymbol{P})$. Immunostaining for LacZ $(\boldsymbol{K}, \boldsymbol{N})$, for OTX2 $(\boldsymbol{L}, \mathbf{0})$, and merge $(\boldsymbol{M}, \boldsymbol{P})$. The white arrowheads indicate both LacZ- and 0TX2-expressing cells $(\boldsymbol{N}-\boldsymbol{P})$. Scale bar, $50 \mu \mathrm{m}$. $\boldsymbol{Q}$, Percentage of 0TX2-negative and 0TX2-positive cells in LacZ-positive cells $(n=418$ cells) in line 1 retinal sections at E15.5. $\boldsymbol{R}$, Structure of EELPOT-Otx2 expression construct. S-U, Immunostaining of retinal sections from WT (S), Otx2 CKO (T), and transgenic mouse line, which expresses Otx2 under the control of EELPOT in the Otx2 CKO background $(\boldsymbol{U})$. Sections were immunostained with an anti-RHODOPSIN antibody (green) and stained with PNA (red) and DAPI (blue) (S-U). Note that EELPOT-directed Otx2 expression in the Otx2 CKO retina resulted in a partial but significant rescue of Otx2 CKO phenotype $(\boldsymbol{U})$. ONL, Outer nuclear layer; INL, inner nuclear layer; GCL, ganglion cell layer. Scale bar, $50 \mu \mathrm{m}$.

We then investigated whether the Otx2 gene driven by EEL$P O T$ can rescue photoreceptor development in the Otx2 CKO retina by generating transgenic mouse line EELPOT-Otx2, which expresses an exogenous mouse Otx2 cDNA under the control of EELPOT (Fig. 4R). We generated three Otx2 $2^{\text {flox/flox}}$; Crx-Cre; EELPOT-Otx2 mouse lines, and examined their retinal photoreceptors at P14. While the Otx $2^{f l o x}$ flox; Crx-Cre mice did not develop retinal photoreceptor cells, including both rods and cones (Nishida et al., 2003), the wild-type mouse retina expresses rhodopsin in rod photoreceptors and PNA in cone photoreceptors at P14 (Fig. 4S). We detected neither RHODOPSIN-positive cells nor PNA-positive cells, in the Otx $2^{\text {floxfflox }}$; Crx -Cre retina (Fig. $4 T)$. In contrast, we observed both RHODOPSIN-positive and PNA-positive cells in the retina in three of three Otx $2^{\text {flox/flox }} ; C r x$ Cre; EELPOT-Otx2 lines (Fig. 4U), although the morphology of the photoreceptor cells was abnormal. Thus, EELPOT-directed Otx2 insufficiently but significantly rescued the photoreceptor development of the Otx2 CKO retina. The partial rescue of pho- toreceptor development is probably due to an insufficient level of and/or improper timing of Otx2 expression in photoreceptor precursors in the EELPOT-Otx2 transgenic retina.

It should be noted that we failed to further narrow down $\sim 500$ bp EELPOT by transgenic mouse assay as far as we attempted, suggesting that EELPOT may function as a unit. Therefore, we next investigated the factors which possibly activate or repress EELPOT based on the cis-elements that appeared in the EELPOT sequence.

\section{Regulation of EELPOT by RAX homeoprotein}

Our observation that OTX2 expression begins in embryonic RPCs mainly during the final cell division (Fig. 1) suggests that the transactivation of Otx2 is regulated by the transcription factor(s) expressed in RPCs and also in postmitotic photoreceptor precursors for a short while. To identify the transcription factor(s) that regulate EELPOT, we searched possible transcription binding sites by a database search, and found paired-type homeo- 
A -16683

5'-GATAGTCTTTTGTTTCCTCTAAATGTCTCAGCC CATGTGGGATAATTAAA

TACAACCCATAAGCAACATTAAGTTTTTAGAGGACCCACAGCTTTAAAT

GCGTGGCTTTGATTTGTACTATAACCTCCATTTTTTTTCCTTTTAAAATTT

GGAAGACAAAATGTGACTTTTCCAACTTACTTAATTTAAAATCACCCCTA

AACACAGGGAAAGAAGCCACTGTAGTGGATGCTGGGGCTGGAGTTGCC TTCATCCCAGCATGCCCCACCACTGTTCTCTGGATGGCAATCACAGGAC CCAGACATCAAGCTGGGGAAGAGATTCTTCCAAGCTTCAG CATCTGGCT CTTTGGCAAACTGAACAGG CAGTTGCCCTGGTGGCTTTAGAGTTGCCTC TGGGTCTGTCACAGGGTATGGCTGCTGACGCAGGATCTGGATTCTTGTC TCGTTTTCATTTCACCATCTTTTTTGGATACCTTTTGGGCATCA-3'
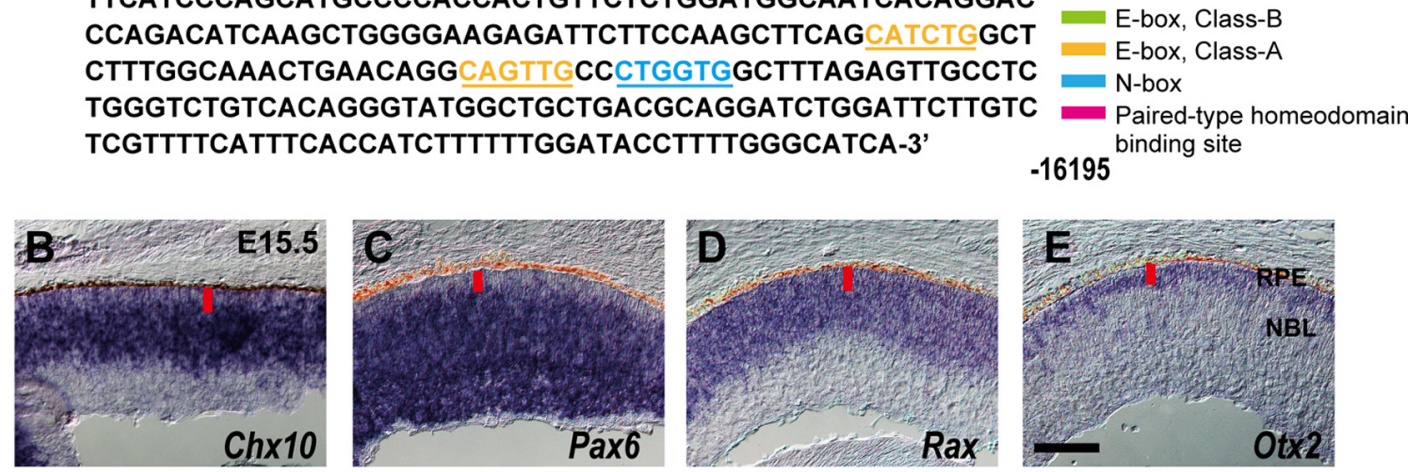

I presumptive photoreceptor layer

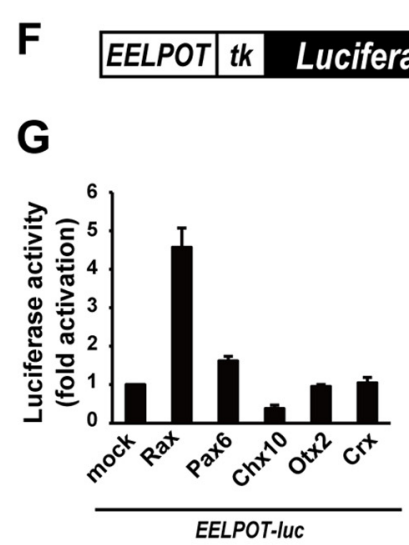

H

$\mathbf{J}$
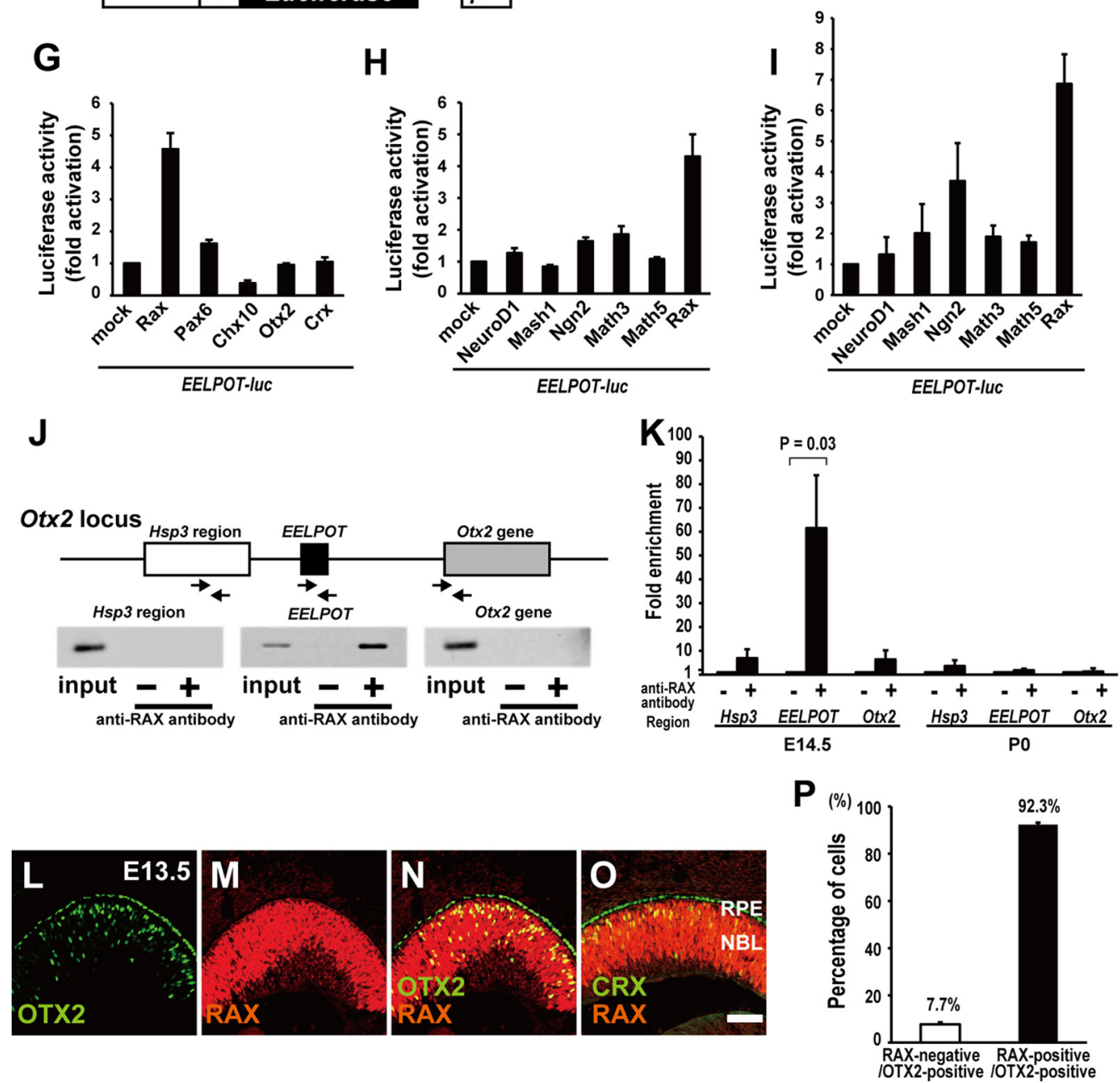

Figure 5. RAX directly regulates Otx2 transcription in the embryonic mouse retina. $A$, DNA sequence of CR1 (EELPOT). Potential paired-type homeodomain-binding site (pink), E-box (class A) (orange), N-box (blue), E-box (class B) (green) are represented. $\boldsymbol{B}$-E, Expression of $C h \times 10(\boldsymbol{B}), \operatorname{Pax} 6(\boldsymbol{C})$, Rax (D), and Otx2 (E) in the developing mouse retina atE15.5. RPE, Retinal pigmentepithelium;NBL, neuroblasticlayer. Scale bar, $100 \mu \mathrm{m}$. $\boldsymbol{F}$, Structure of EELPOT-luc reporter construct. $\mathbf{G}-\mathbf{I}$, Luciferase reporter assay using EELPOT-luc. NIH3T3 cells $(\mathbf{G}, \boldsymbol{H})$ and Neuro2A $(\boldsymbol{I})$ cells were transfected with EELPOT-luc reporter construct and expression plasmids of homeodomain transcription factors $(G)$ or bHLH transcription factors $(\boldsymbol{H}, \boldsymbol{I})$. Data are means \pm SEM $(n=3)$.J, Mouse Otx2 locus and primers for $P C R$ of ChIP assay. The arrows represent primers for PCR. Mouse retinas at E15.5 were harvested and subjected to ChIP assay using the anti-RAX antibody. PCR was performed using primer set designed to 5 '-upstream region of EELPOT (Hsp3 region), EELPOT, or OtX2 gene. $\boldsymbol{K}$, Quantitative ChIP analysis both E14.5 and PO for anti-RAX antibody. Data are means \pm SEM $(n=4)$. Values of $p$ by Student's $t$ test. $\boldsymbol{L}-\mathbf{0}$, Immunostaining for 0 TX2 ( $\boldsymbol{L}$ ) and RAX ( $\boldsymbol{M}$ ). ( $(\boldsymbol{M})$, Merge of $(\boldsymbol{L})$ and $(\mathbf{M})$. Coimmunostaining for RAX and CRX (0). Scale bar, $100 \mu \mathrm{m}$. P, Percentage of RAX-positive cells in 0TX2-positive cells. $n=195$ cells from 3 sections were analyzed. Data are means \pm SEM. 
A

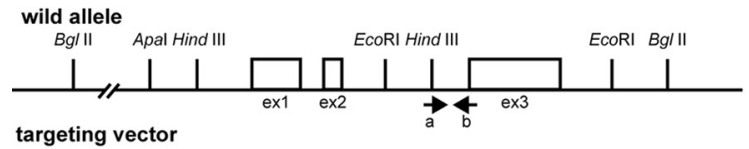

targeting vector

Bgl II Apal Hind III EcoRIHind III Hind III

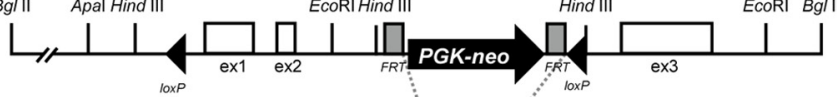

Rax flox allele

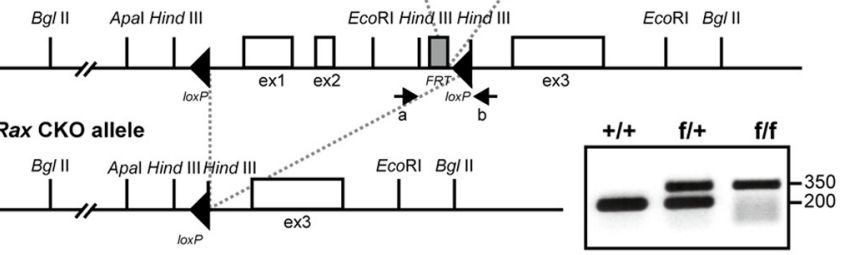

B

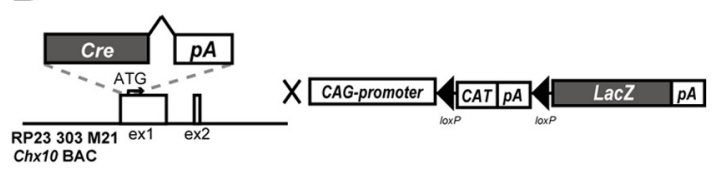

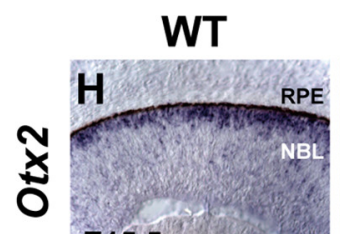

Rax CKO

E15.5
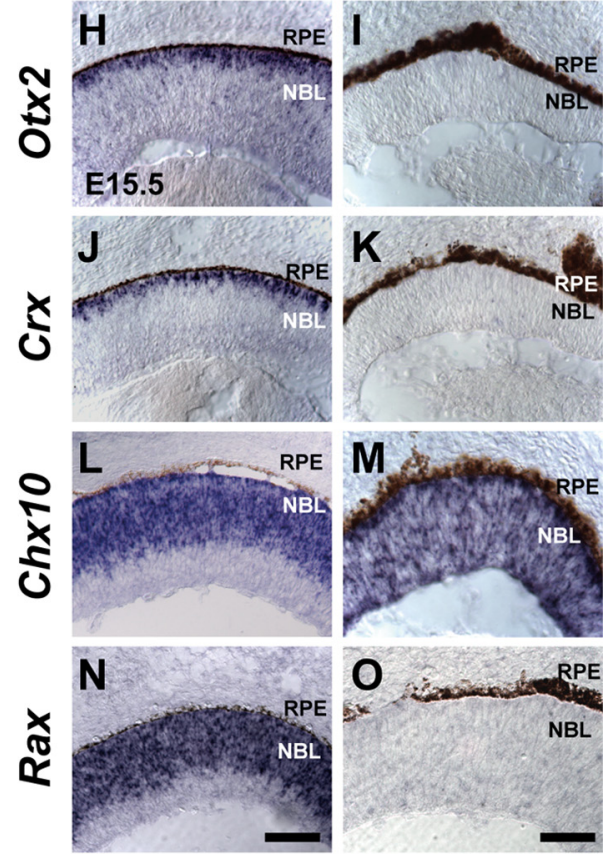
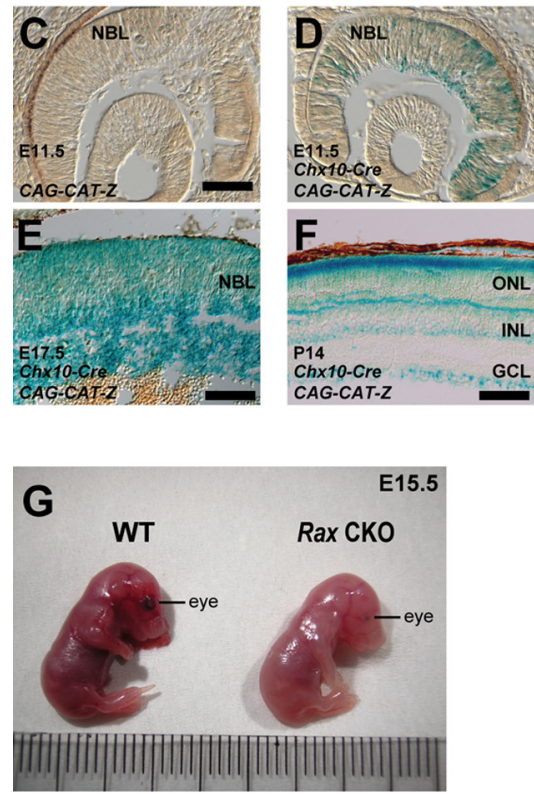

$\mathbf{P}$

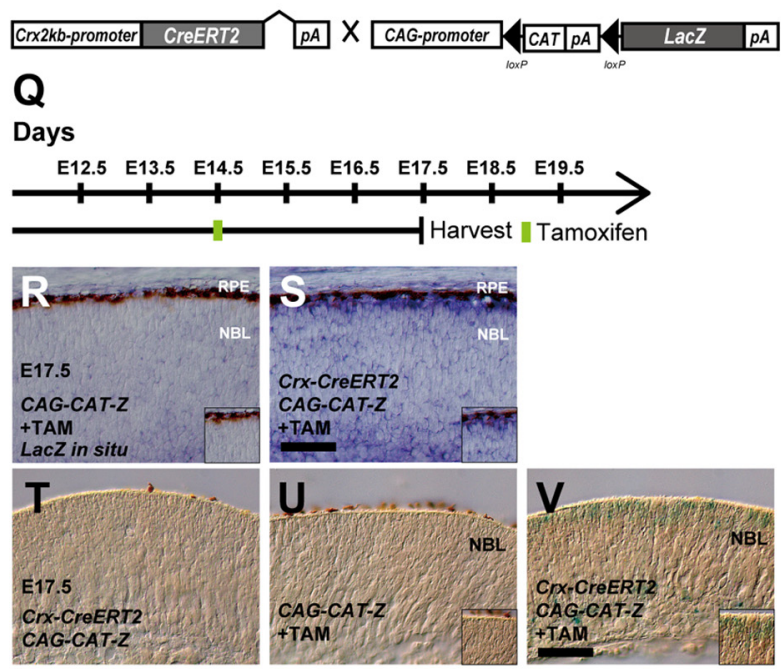

W

Days

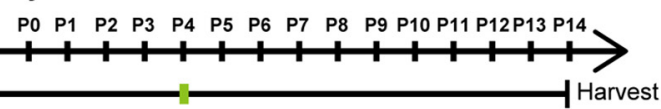

Tamoxifen
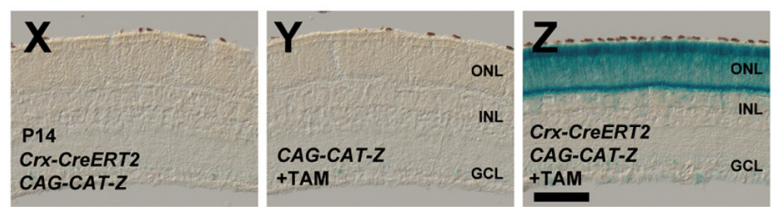

Figure 6. Establishment and analysis of Rax flox, Crx 2 kb promoter-CreERT2, and Chx10-Cre mouse lines. A, Diagram of the Rax WT allele, the targeting vector, Rax flox allele, and Rax CKO allele. The white boxes, gray boxes, and arrowheads indicate exons, FRT sites, and loxP sites, respectively. PGK-neo indicates a phosphoglycerate kinase (PGK) promoter-driven neomycin-resistant gene expression cassette. The arrows indicate the positions of PCR primers used for genotyping. PCR products of 200 or 350 bp were amplified from WT or targeted allele, respectively. $\boldsymbol{B}$, A schematic diagram of the modified BAC integrated with the Cre-pA cassette into the first exon of the mouse Chx10 gene, and CAG-promoter-directed LacZ construct. LacZ expression starts after the recombination occurs at loxP sites flanking chloramphenicol acetyltransferase (CAT)-pA cassette (CAG-CAT-Z). C-F, X-gal staining using sections from the retina of CAG-CAT-Z mouse at E11.5 (C) and from the retina of Chx10-Cre; CAG-CAT-Z mouse of E11.5 (D), E17.5 (E), or P14 (F). Scale bars: C, $200 \mu \mathrm{m} ; \boldsymbol{E}, 50 \mu \mathrm{m} ; \boldsymbol{F}, 100 \mu \mathrm{m}$. NBL, Neuroblastic layer; ONL, outer nuclear layer; INL, inner nuclear layer; GCL, ganglion cell layer. G, A picture showing mouse embryos at E15.5, WT (left) or Chx10-Cre; Rax ${ }^{\text {flox/flox }}$ (Rax CKO) mouse (right). $\boldsymbol{H}-\mathbf{O}$, In situ hybridization of sections from the control

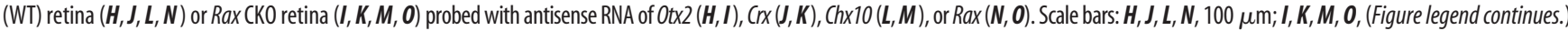


protein binding sites (Wilson et al., 1993), E-box (class A and class B) and N-box motifs in EELPOT (Fig. 5A). Several pairedtype homeoproteins expressed in RPCs are known, including CHX10, PAX6, and RAX (Levine and Green, 2004). We compared the expression patterns of Chx10, Pax6, Rax, and Otx2 in the embryonic retina at E15.5 by in situ hybridization to identify which factor is expressed both in progenitors and developing photoreceptor precursors (Fig. 5B-E). Both Rax and Otx2 expression were observed in the presumptive photoreceptor layer, while Chx10 and Pax6 were expressed in RPCs but weakly observed in the outermost region of the retina at late embryonic stages (Fig. 5B-E). Thus, we investigated the possibility that RAX may transactivate EELPOT by luciferase assay using NIH3T3 cells. We observed that RAX significantly transactivated a luciferase reporter in which EELPOT was linked with thymidine kinase minimal promoter and the luciferase gene (EELPOT-luc), while PAX6 and CHX10 did not significantly transactivate EELPOT (Fig. $5 F, G$ ). bHLH factors, NEUROD1, MASH1, and NEUROGENIN2 (NGN2), can bind to E-box and have been implicated in photoreceptor fate determination and/or photoreceptor differentiation. This raises the possibility that EELPOT is regulated by bHLH transcription factors. We observed that NGN2 and MATH3 activated EELPOT-luc reporter expression only very weakly, and neither NEUROD1 nor MASH1 significantly activated EELPOT-luc (Fig. 5H). In addition, we performed luciferase assay using a neuronal cell line, Neuro2A. We observed that RAX significantly activated EELPOT as observed in NIH3T3 cells. It should be noted that we observed weak activation of EELPOT by NGN2 (Fig. 5I). However, photoreceptor development was not significantly affected in either $\mathrm{Ngn} 2^{-/-}$; Ascl1 $1^{-/-}$; Math $3^{-/-}$or $\mathrm{Ngn} 2^{\text {GFP/GFP }}$ mutant retinas (Akagi et al., 2004; Hufnagel et al., 2010), suggesting that NGN2 does not play a critical role in regulating EELPOT. These results suggest that EELPOT can be directly regulated by RAX homeoprotein.

We next tested whether RAX binds to EELPOT by ChIP assay (Fig. 5J). We performed a ChIP assay using E15.5 mouse retinal lysate with an anti-RAX antibody. We PCR-amplified three distinct genomic regions with primers corresponding to the $5^{\prime}$ upstream region of EELPOT (Hsp3 region), to EELPOT, and to Otx 2 coding region (Otx2 gene) following immunoprecipitation (Fig. $5 J$ ). We observed a significant amplified band only with the primers for EELPOT, suggesting that RAX binds to EELPOT to transactivate $O t \times 2$ in the embryonic mouse retina. However, we did not observe EELPOT activation by RAX in the P0 retina (Fig. $4 G)$. We, therefore, examined whether RAX binds to EELPOT in the $\mathrm{P} 0$ retina. We did not observe a significant RAX binding to EELPOT (Fig. 5K), consistent with the observation that EELPOT was inactive at P0. We then examined whether RAX and OTX2 are coexpressed in photoreceptor precursors by immunostaining at E13.5. We observed that almost all OTX2-expressing cells are

$\leftarrow$

(Figure legend continued.) $50 \mu \mathrm{m} . \boldsymbol{P}$, Structure of $\mathrm{Cr} \times 2 \mathrm{~kb}$-promoter-driven CreERT2 construct and CAG-CAT-Z construct. $\boldsymbol{Q}$, Schedule for tamoxifen injection and harvest of embryos. $\boldsymbol{R}-\boldsymbol{V}$, In situ hybridization probed with an antisense RNA against $\operatorname{LacZ}(\boldsymbol{R}, \mathbf{S})$ or X-gal staining $(\boldsymbol{T}-\boldsymbol{V})$ of sections isolated from a Crx-CreERT2; CAG-CAT-Z mouse retina without tamoxifen ( $\boldsymbol{T})$ and CAG-CAT-Z mice ( $\boldsymbol{R}, \boldsymbol{U})$ or Crx-CreERT2; CAG-CAT-Z mice $(\boldsymbol{S}, \boldsymbol{V})$ with tamoxifen. Tamoxifen was administrated to pregnant female mice at E14.5, and embryos were harvested at E17.5 as indicated in Q. The insets indicate the representative position of each panel. Scale bar, $50 \mu \mathrm{m}$. $W-Z, X$-gal staining using sections from a CrX-CreERT2; CAG-CAT-Z mouse retina without tamoxifen $(\boldsymbol{X})$ and CAG-CAT-Z mice ( $\boldsymbol{V}$ ) or CrX-CreERT2; CAG-CAT-Z mice (Z) with tamoxifen. Tamoxifen was administrated to mice at P4, harvested at P14 as indicated in $(\boldsymbol{W})$, and subsequently processed for X-gal staining. Scale bar, $100 \mu \mathrm{m}$. positive for RAX (Fig. $5 L-N, P$ ), consistent with the observation that RAX transactivates EELPOT. Similarly, we observed coexpression of RAX and CRX in differentiating photoreceptor precursors at E13.5 (Fig. 5O).

We tried to identify a RAX-binding site(s) in EELPOT; however, we could not narrow down EELPOT into a smaller region activated by RAX using a luciferase assay as far as we attempted. There may be multiple atypical RAX-binding sites that have a low affinity to RAX in EELPOT. This result may be consistent with our inability to narrow down EELPOT by transgenic mouse assay.

\section{RAX is required for Otx 2 expression in early photoreceptor precursors}

We inactivated Rax by conditional targeted gene disruption to examine whether RAX is required for Otx2 expression in the developing retina in vivo (Fig. $6 A-O$ ). We established a transgenic mouse line harboring a $\mathrm{BAC}$ containing a Cre recombinase gene immediately before the site corresponding to the CHX10 initiation codon, and named this transgenic mice line Chx10-Cre (Fig. $6 B)$. This transgenic mouse line expresses Cre in RPCs, and recombination is detected from E11.5 in the retina by mating with the CAG-CAT-Z Cre monitor mouse line (Fig. 6B-F). We generated Rax flox/flox; Chx10-Cre mice, which we referred to as Rax CKO mice, and examined Otx2 expression in the E15.5 retina. Since Rax is essential for RPC proliferation (Mathers et al., 1997), the size of the Rax CKO retina was significantly smaller than that of the WT retina (Fig. 6G). Otx2 mRNA is expressed mainly in the presumptive photoreceptor layer at E15.5. We observed a severe attenuation of Otx2 expression in the Rax CKO retina (Fig. $6 H, I$ ), in which the Rax gene was inactivated in RPCs (Fig. $6 N, O)$. Crx , a downstream target gene of OTX2, was also strongly reduced (Fig. $6 \mathrm{~J}, \mathrm{~K})$. In contrast, we observed almost normal or slightly enhanced expression of Chx10 in the Rax CKO retina compared with that in the WT retina (Fig. $6 L, M$ ). We, therefore, could not exclude the possibility that Rax inactivation in the progenitor cells affects internal properties of RPCs, resulting in the severe attenuation of Otx2 and Crx expression in the Rax $\mathrm{CKO}$ retina by an indirect effect of Rax inactivation. To exclude this possibility, we inactivated the Rax gene in the early postmitotic photoreceptor precursors using the CreERT2 system. To attain a tight regulation of Cre expression in photoreceptor precursors, we established a transgenic mouse line, referred to as Crx-CreERT2 that expresses CreERT2 under the control of the 2 kb Crx promoter (Fig. 6P). We confirmed that application of tamoxifen to this mouse line induced Cre-mediated recombination of a LacZ reporter transgene in the developing photoreceptor layer (Fig. 6Q-Z)

We then generated Rax flox/flox; Crx-CreERT2 mice and treated them with tamoxifen at E14.5 to inactivate Rax in embryonic photoreceptor precursors (Fig. 7A). We referred to this mouse as the Rax-induced CKO (Rax iCKO) mouse in this study. To examine whether induced Rax inactivation occurs, we analyzed E15.5 retinas by in situ hybridization. We observed a loss of Rax expression in the presumptive photoreceptor layer of the retina corresponding to the $C r x$-expressing region (Fig. $7 B-E$ ). We observed that Otx2 expression was severely attenuated in the Rax iCKO retina, while Pax6 expression was barely affected and Chx10 expression was slightly upregulated in the presumptive photoreceptor layer (Fig. 7F-Q). These results suggest that Rax is required for normal Otx2 expression in embryonic photoreceptor precursors.

We previously reported that the Otx2-deficient retina showed a total loss of photoreceptors and a remarkable increase of Pax6- 


\section{A Days}
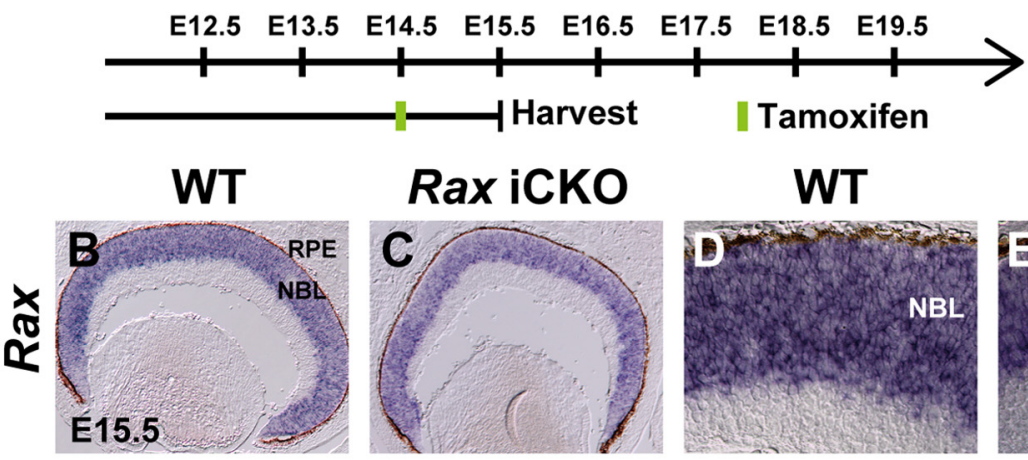

Rax ICKO
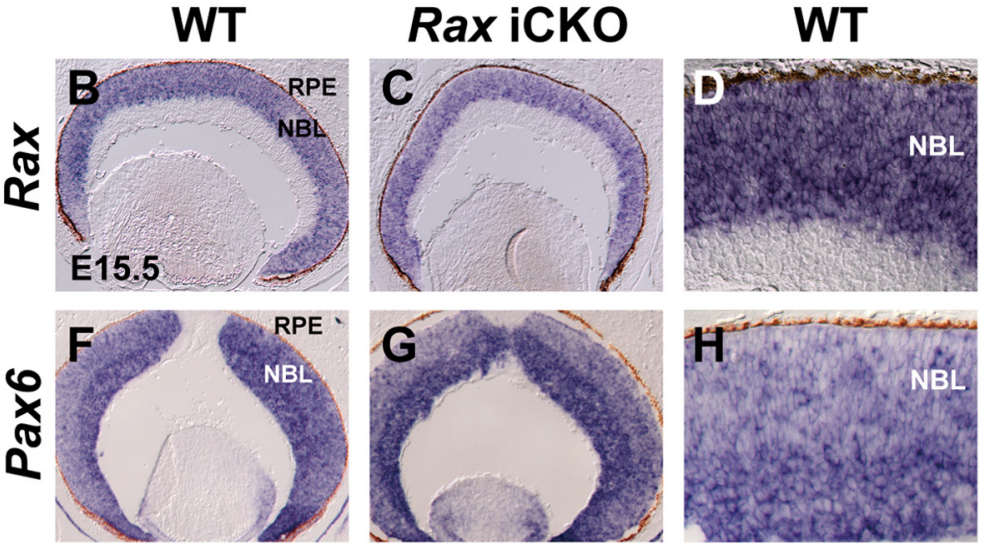

Rax ICKO
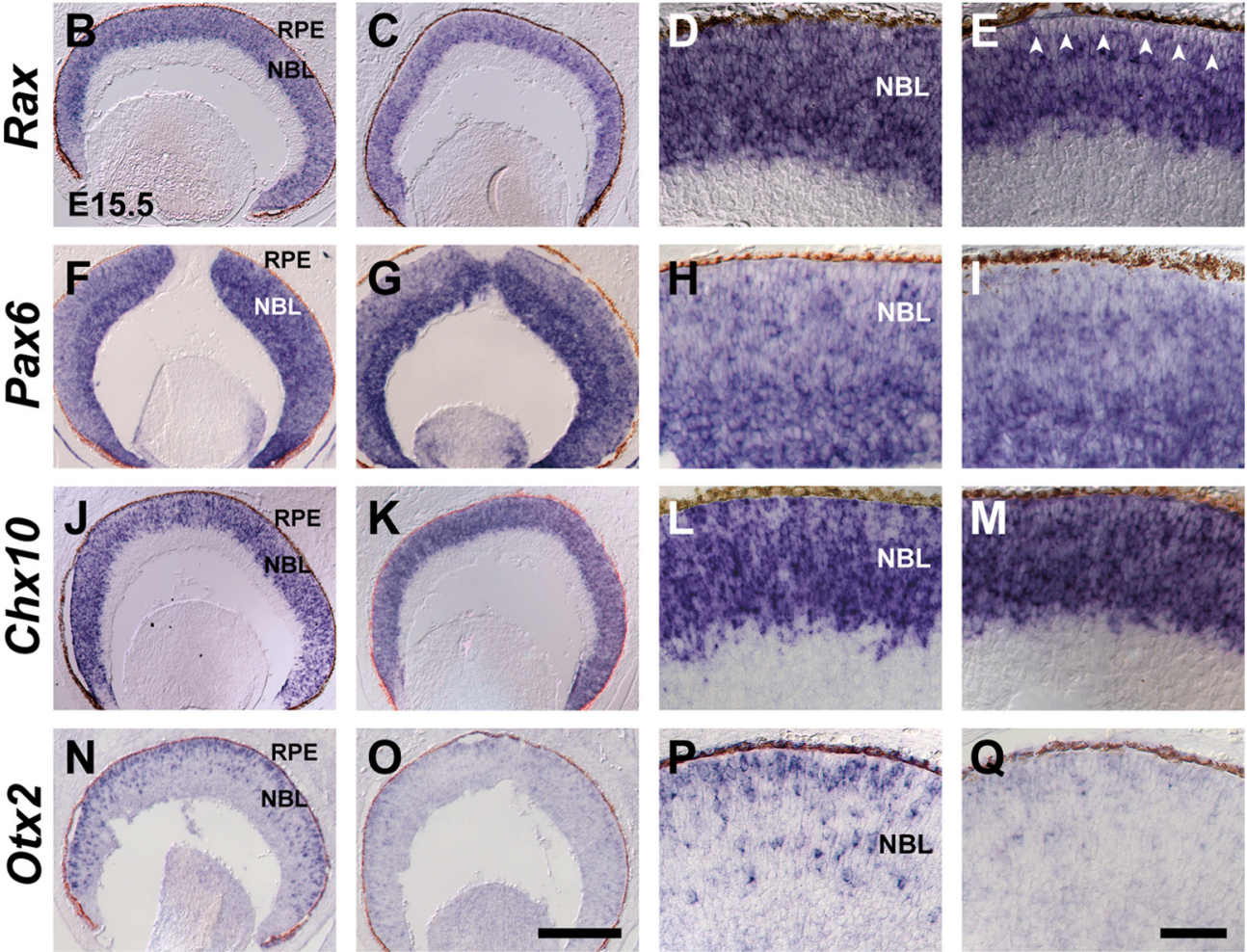

Days in vitro (DIV)

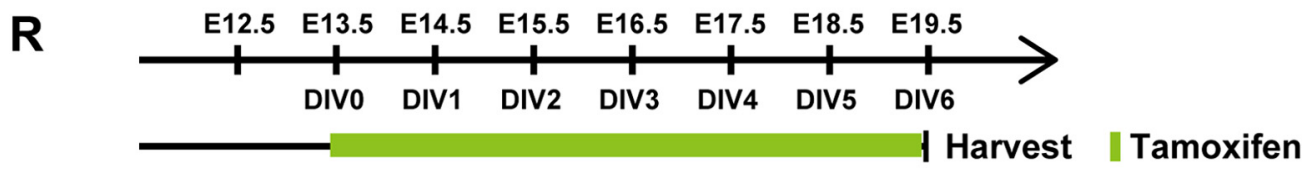

S
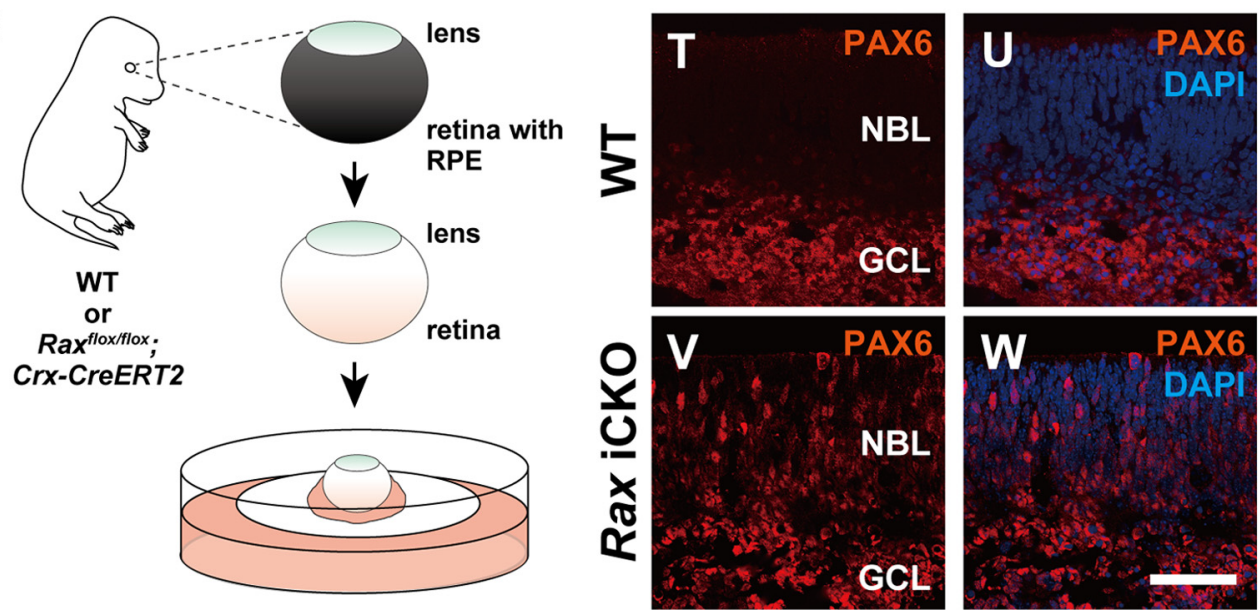

Figure 7. Immunohistological analysis of the tamoxifen-induced Rax CKO (iCKO) retina. $\boldsymbol{A}$, Schematic diagram of the schedule for tamoxifen injection and harvest of embryos. $\boldsymbol{B}-\mathbf{Q}$, In situ hybridization of sections from the control (WT) retina $(\boldsymbol{B}, \boldsymbol{D}, \boldsymbol{F}, \boldsymbol{H}, \boldsymbol{J}, \boldsymbol{L}, \boldsymbol{N}, \boldsymbol{P})$ or RaxiCKO retina $(\boldsymbol{C}, \boldsymbol{E}, \mathbf{G}, \boldsymbol{I}, \boldsymbol{K}, \boldsymbol{M}, \mathbf{O}, \mathbf{Q})$ probed with antisense RNA of $\operatorname{Rax}(\boldsymbol{B}-\boldsymbol{E}), \operatorname{Pax} 6(\boldsymbol{F}-\boldsymbol{I})$, Chx10(J-M), and 0 tx2 (N-Q). $D, E ; H, I ; L, M$; and $\boldsymbol{P}, \boldsymbol{Q}$ are higher magnifications of $\boldsymbol{B}, \boldsymbol{C} ; \boldsymbol{F}, \boldsymbol{G} ; \boldsymbol{J}, \boldsymbol{K} ;$ and $\boldsymbol{N}, \boldsymbol{O}$, respectively. The white arrowheads indicate the region where Rax gene was inactivated by tamoxifen-induced recombination (E). RPE, Retinal pigment epithelium; NBL, neuroblastic layer. Scale bars: $\mathbf{0}, 200 \mu \mathrm{m} ; \mathbf{Q}, 50 \mu \mathrm{m}$. $\boldsymbol{R}$, Schematic diagram of the schedule for tamoxifen treatment and harvest of explant cultures. $\boldsymbol{S}$, E13.5 mouse retinas were used for explant culture after removal of RPE. Note that lenses were not removed. $\boldsymbol{T}-\boldsymbol{W}$, Immunostaining for PAX6 of the WT $(\boldsymbol{T}, \boldsymbol{U})$ or Rax iCKO $(\boldsymbol{V}, \boldsymbol{W})$ retina. Images of PAX6 immunostaining without $(\boldsymbol{T}, \boldsymbol{V})$ or with $(\boldsymbol{U}, \boldsymbol{W})$ DAPI counterstaining. GCL, Ganglion cell layer; NBL, neuroblastic layer. Scale bar, $50 \mu \mathrm{m}$. 
A

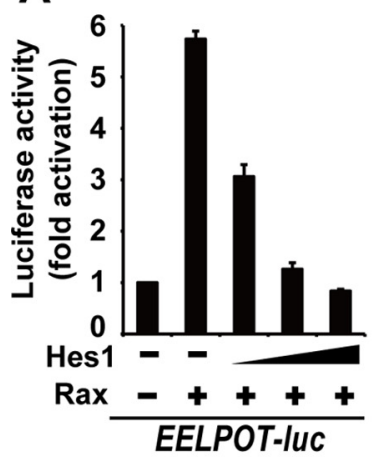

D

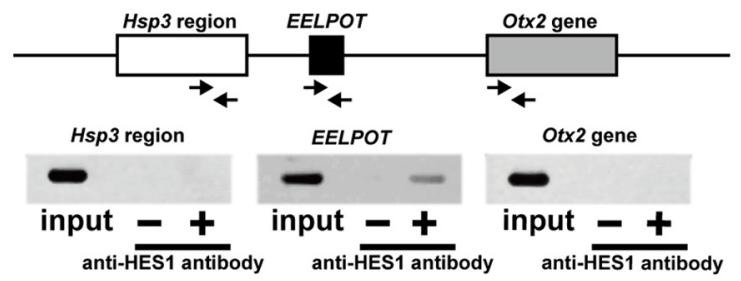

B

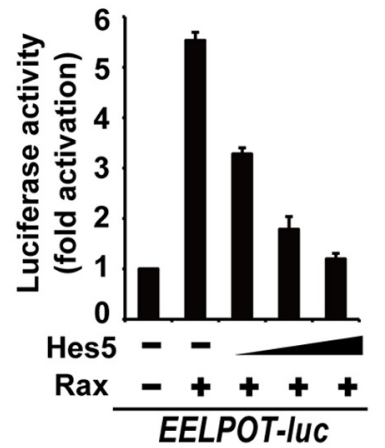
EELPOT-IUC Hes5 - -

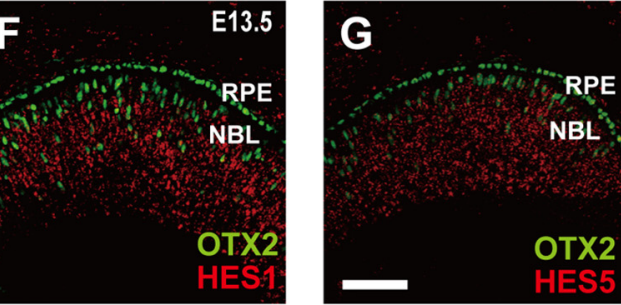

J

Days in vitro (DIV)

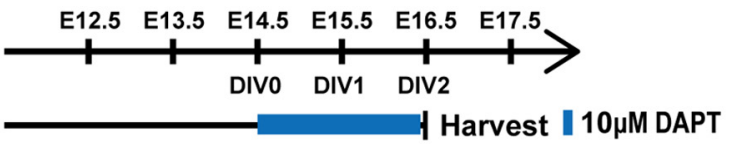

H

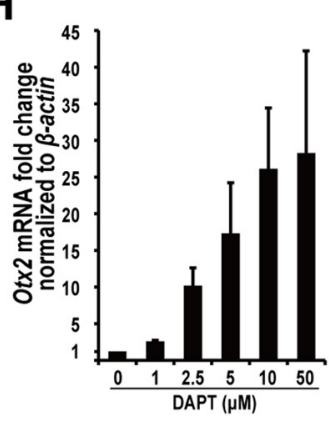

I

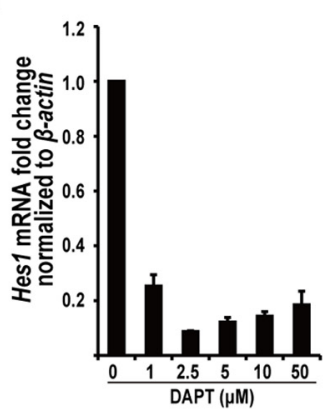

\section{$\mathbf{N}$}

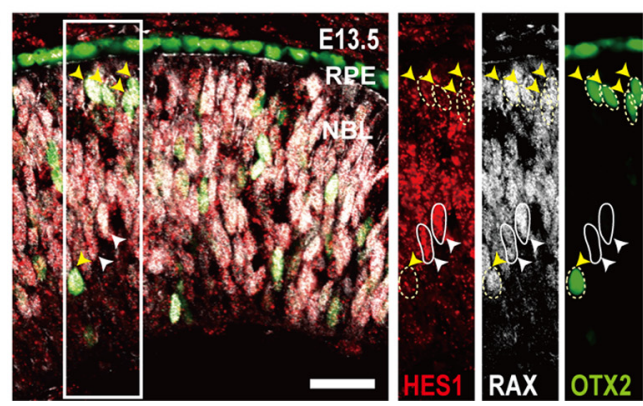

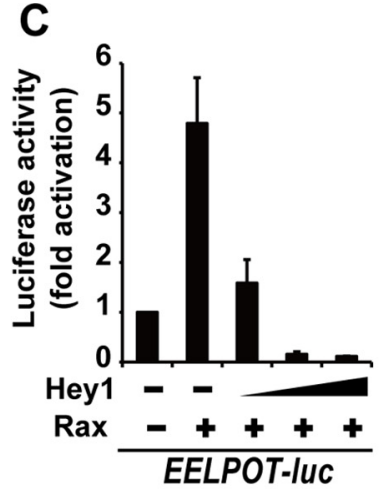

E

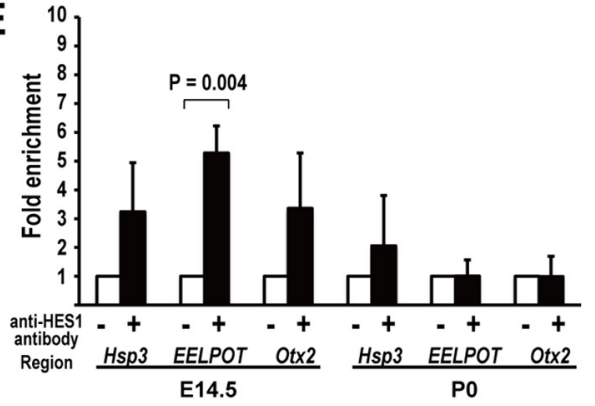

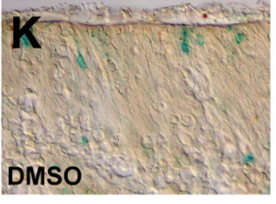
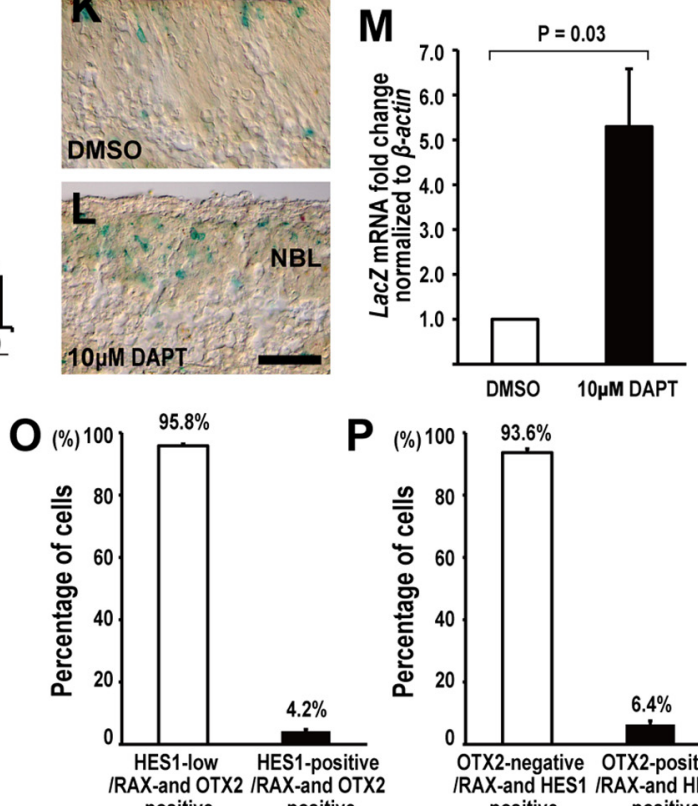

-positiver -positive

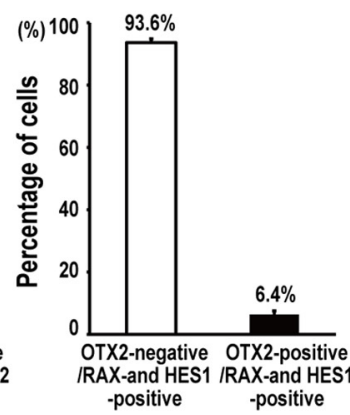

Figure 8. HES family proteins repress Otx2 transcription. $A-C$, Luciferase reporter assay using EELPOT-luc. NIH3T3 cells were transfected with EELPOT-luc reporter construct plus Rax expression plasmid $(0.5 \mu \mathrm{g})$ and Hes 1 expression plasmid $(0.1,0.2,0.5 \mu \mathrm{g})(\boldsymbol{A})$, or EELPOT-luc reporter construct plus Rax expression plasmid and Hes5 expression plasmid $(0.1,0.2,0.5 \mu \mathrm{g})(\boldsymbol{B})$, or EELPOT-luc reporter construct plus Rax expression plasmid and Hey1 expression plasmid $(0.1,0.2,0.5 \mu \mathrm{g})(\boldsymbol{C})$. Data are means \pm SEM $(n=3)$. D, Mouse Otx2 locus and primers for PCR of ChIP assay. The arrows represent primers for PCR. Mouse retinas at E14.5 were harvested and subjected to ChIP assay using an anti-HES1 antibody. PCR was performed using primer set designed to $5^{\prime}$-upstream region of EELPOT (Hsp3 region), EELPOT, or Otx2 gene. E, Quantitative ChIP analysis both E14.5 and PO for anti-HES1 antibody. Data are means \pm SEM ( $n=4$ ). Values of $p$ by (Figure legend continues.) 
positive amacrine-like neurons (Nishida et al., 2003). We, therefore, examined whether the Rax iCKO retina showed an increase of Pax6-positive amacrine-like neurons as observed in the Otx2 CKO retina. Cell fate determination of amacrine cells mainly occurs at late embryonic stages. Since the embryos did not survive until the late embryonic stages when pregnant females received tamoxifen, we performed a retinal explant culture using the E13.5 Rax flox/flox $_{\text {; }}$ Crx-CreERT2 retina (Fig. $7 R, S$ ). Retinal explants were cultured in tamoxifen-containing medium for $6 \mathrm{~d}$ and subjected to immunostaining (Fig. $7 T-W$ ). We observed Pax6-positive cells, which marks RPCs and amacrine cells, at this stage, in the region where RPCs and amacrine cells reside in the control retina (Fig. 7T,U). In contrast, in the Rax iCKO retina, we observed a significant number of ectopic Pax6-positive cells across the retina (Fig. $7 V, W)$.

\section{HES family repressors antagonize the RAX activity on EELPOT}

In EELPOT, one N-box, a HES family protein-binding sequence, and three E-boxes overlapping with class A or class B bHLH transcription factor-binding sequences are present (Iso et al., 2003) (Fig. 5A). RAX and HES proteins are implicated in the maintenance of RPCs, whereas bHLH transcription factors appear to induce neuronal differentiation (Levine and Green, 2004; Ohsawa and Kageyama, 2008). To investigate whether HES family proteins affect RAX-dependent reporter gene activation, we performed a luciferase assay using NIH3T3 cells. We focused on HES1, HES5, and HEY1/HESR1. These molecules function downstream of the NOTCH pathway (Jadhav et al., 2006; Fischer and Gessler, 2007; Ohsawa and Kageyama, 2008) and are significantly expressed in the embryonic retina but downregulated in FACS-sorted postmitotic Crx-promoter-driven-EGFP-positive photoreceptor precursor cells (data not shown) (Muranishi et al., 2010). These observations raised the possibility that HES1, HES5, or HEY1 act on EELPOT in RPCs but are downregulated in postmitotic cells. We cotransfected a Hes1, Hes5, or Hey1 expression plasmid together with a Rax expression plasmid and an EELPOT reporter plasmid. We observed that HES1, HES5, and HEY1 suppressed RAX-induced luciferase activation in a dose-dependent manner (Fig. $8 A-C$ ). Next, we performed a ChIP assay using E14.5 mouse retinal lysate with an anti-HES1 antibody (Fig. $8 D$ ). We observed a significant amplified band only with the primers for EELPOT, suggesting that HES1 is directly involved in regulating Otx2 transcription through EELPOT at E14.5 (Fig. $8 D$ ). We next examined whether HES1 binds to EELPOT in the P0 retina

\section{$\leftarrow$}

(Figure legend continued.) Student'st test. $F, G$, Coimmunostaining for OTX2 and HES1 $(\boldsymbol{F})$, or for 0TX2 and HES5 (G). RPE, Retinal pigment epithelium; NBL, neuroblastic layer. Scale bar, 100 $\mu \mathrm{m} . \boldsymbol{H}, \boldsymbol{I}$, A relative expression level of 0 tx2 $(\boldsymbol{H})$ or Hes $7(\boldsymbol{I})$ mRNA normalized to $\beta$-actin mRNA in the organ-cultured retina from E13.5 to E15.5 in the presence of DAPT by quantitative RT-PCR analysis. Data are means \pm SEM $(n=3) . J-M$, Schematic diagram of the schedule for DAPT treatment and harvest of explant cultures (J). X-gal staining of the EELPOT-LacZ without DAPT $(\boldsymbol{K})$ or with DAPT $(\boldsymbol{L})$ retina. A relative expression level of $L a c Z(\boldsymbol{M})$ mRNA normalized to $\beta$-actin mRNA in the organ-cultured EELPOT-LacZ mouse retina from E14.5 to E16.5 in the presence of DAPT $(10 \mu \mathrm{M})$ by quantitative RT-PCR analysis. Data are means $\pm \operatorname{SEM}(n=3)$. Values of $p$ by Student's $t$ test. Scale bar, $20 \mu \mathrm{m}$. $N$, Immunostaining for HES1, RAX, and OTX2. The white arrowheads indicate RAX-positive, HES1-positive, and OTX2-negative cells delineated by circle. The yellow arrowheads indicate RAX-positive, OTX2-positive, and HES1-low cells delineated by dotted circle. Scale bar, $50 \mu \mathrm{m} . \mathbf{0}$, Percentage of HES1-positive or -low cells in RAX and 0TX2 double-positive cells. Data are means \pm SEM. $n=239$ cells from 3 sections were analyzed. $\boldsymbol{P}$, Percentage of OTX2-positive or -negative cells in RAX and HES1 double-positive cells. Data are means \pm SEM. $n=425$ cells from 3 sections were analyzed. and did not observe any significant HES1 binding to EELPOT (Fig. $8 E$ ), suggesting that HES1 binding is not required for the suppression of EELPOT at P0, consistent with the observation that RAX did not bind to EELPOT at P0 (Fig. $5 \mathrm{~K}$ ).

We then performed immunostaining of OTX2 and HES in the developing mouse retina at E13.5, and observed that most of the OTX2-positive cells were HES1 and HES5 negative (Fig. $8 F, G$ ), supporting the idea that HES proteins negatively regulate Otx2 transcription. However, RAX and HES proteins are expressed in proliferating RPCs.

HES family molecules function downstream of RBPJ in the canonical NOTCH signaling pathway. It was reported that $R B P j$ inactivation using another Chx10-Cre transgenic line showed significant upregulation of Otx 2 and $C r x$ in the developing retina at E13.5 and E16.5 by in situ hybridization (Zheng et al., 2009). In addition, we observed significant upregulation of Otx2 transcription in the organ-cultured embryonic retina from E13.5 to E15.5 in the presence of DAPT, a NOTCH signal inhibitor (Fig. $8 \mathrm{H}, \mathrm{I}$ ). We further investigated whether EELPOT is suppressed by $\mathrm{NOTCH}$ signaling using organ-cultured embryonic retinas isolated from EELPOT (CR1)-LacZ transgenic mice (Fig. 8J). We observed a significant increase in the number of LacZ-positive cells in the retina treated with DAPT compared with that in the DMSO-treated retina (Fig. $8 K, L$ ). We also detected a significant increase in the amount of $L a c Z$ mRNA in the DAPT-treated retina (Fig. $8 \mathrm{M}$ ). These results support our hypothesis that EELPOT is directly suppressed by the NOTCH-HES canonical pathway in the developing retina.

We further investigated RAX, OTX2, and HES1 expression in the embryonic mouse retina by immunostaining to examine whether both RAX- and OTX2-expressing cells express HES1 (Fig. 8N-P). Most RAX and OTX2 double-expressing cells express notably low levels of HES1 (Fig. $8 \mathrm{~N}, \mathrm{O}$ ), supporting the idea that RAX is also expressed in postmitotic photoreceptor precursors as well as in mitotic RPCs. We also investigated whether both RAX- and HES1-expressing cells express OTX2. Most RAX- and HES1-expressing cells did not express OTX2 (Fig. 8N,P), suggesting that activation of $O t x 2$ transcription by RAX requires the downregulation of HES family proteins, consistent with our observations obtained by luciferase reporter assay (Fig. $8 \mathrm{~A}-\mathrm{C}$ ).

We further examined the effect of HES1 on Otx2 expression in vivo using transgenic mice. We generated transient transgenic mice using the Crx promoter-driven $L a c Z$ control construct or the Crx promoter-driven Hesl construct (Fig. 9A,F), and examined their retinas by immunostaining at E13.5 (Fig. 9B-D, $G-I$ ). While most LacZ-expressing cells overlapped with OTX2expressing cells (Fig. 9B-E), most HES1-expressing cells did not express OTX2 (Fig. 9G-J), suggesting that HES proteins can suppress Otx 2 transcription even in postmitotic photoreceptor precursors by forced expression, because the Crx promoter is active in the postmitotic photoreceptor precursors. HES family protein-mediated $\mathrm{Otx} 2$ repression appears to be consistent with the observation that OTX2 expression begins in the final cell cycle of RPCs.

\section{Discussion}

Otx2 expression mechanism in embryonic photoreceptor cell fate determination

In the current study, we first observed that OTX2 protein expression begins mainly in the final cell cycle of RPCs (Fig. 1). Expression patterns are similar between Otx2 transcripts detected by in situ hybridization (Fig. 2B) and OTX2 protein detected by immunostaining (Fig. $5 L$ ) in the E13.5 retina, indicating that OTX2 

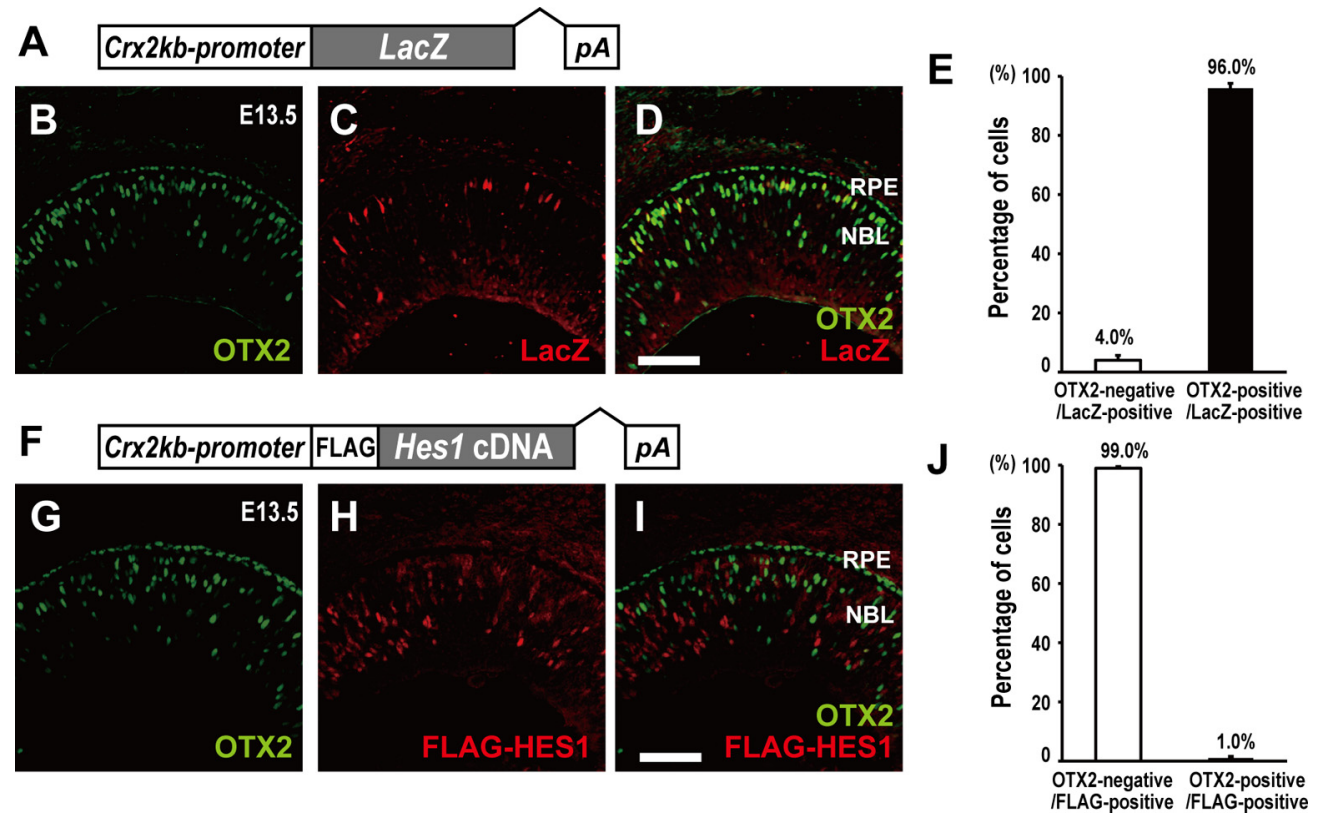

Figure 9. Effect of HES1 on Otx2 expression in vivo. $\boldsymbol{A}$, Structure of $(r \times 2 \mathrm{~kb}$-promoter-directed LacZ construct. B-D, Immunostaining for OTX2 (B) and LacZ (C). (D), Merge of (B) and (C). The presumptive photoreceptor layer lies between the RPE and NBL. Scale bar, $100 \mu \mathrm{m}$. E, Percentage of OTX2-positive or -negative cells in LacZ-positive cells. Data are means \pm SEM. $n=199$ cells on 12 sections of 3 retinas from 3 animals were analyzed. $\boldsymbol{F}$, Structure of $(r \times 2$ kb-promoter-directed FLAG-Hes 1 construct. $\mathbf{G}-\boldsymbol{I}$, Immunostaining for 0TX2 (G) and FLAG-HES1 (H). (I), Merge of ( $(\boldsymbol{G})$ and (H). Scale bar, $100 \mu \mathrm{m}$. J, Percentage of OTX2-positive or -negative cells in FLAG-HES1-positive cells. Data are means \pm SEM. $n=298$ cells on 4 sections of 3 retinas from 3 animals were analyzed.

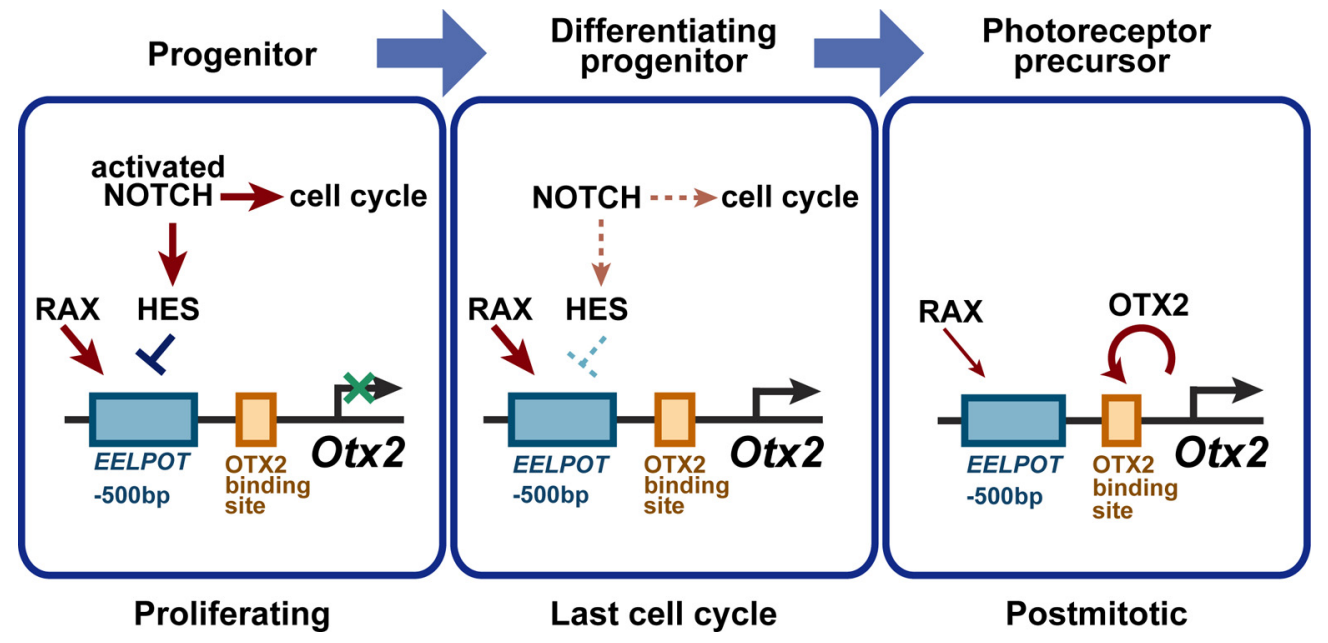

Figure 10. A hypothetical model of the regulatory mechanism of Otx2 expression in embryonic photoreceptor cell fate determination. In proliferating RPCS, the EELPOT enhancer is suppressed by HES family protein(s), and suppression of Otx2 transcription is maintained. Activated NOTCH signaling stimulates cell proliferation. In the last RPC cell cycle, the NOTCH-HES signal turns off or significantly decreases, and RAX acts on EELPOT to transactivate Otx2. RAX steers an undifferentiated progenitor cell, which is in its final cell cycle, to the photoreceptor cell fate by transactivating Otx2. In postmitotic precursors, RAX is expressed for at least some period and continuously activates 0 tx2. 0TX2 transactivates its own transcription through multiple 0TX2-binding sites and various downstream genes including Crx encoding a transcription factor required for maturation of photoreceptors.

protein is expressed soon after Otx2 transcription. We then identified an evolutionarily conserved $O t \times 2$ retinal enhancer, EELPOT, which is essential for Otx2 transcription in embryonic photoreceptor precursors. Our results showed that EELPOT is an Otx2 enhancer distinct from the Otx2 brain enhancers (Kurokawa et al., 2004a,b) and directs Otx2 expression in the embryonic retina.

Although Otx2 is required both for cone cell fate determination during embryonic stages and rod cell fate determination mainly at postnatal stages, EELPOT did not appear to direct reporter gene transcription after $\mathrm{P} 0$ in the transgenic mouse retina (Fig. $4 F, G$ ), suggesting that EELPOT mainly functions to initiate the Otx2 expression required for embryonic photoreceptor cell fate determination but not for the maintenance of Otx2 expression in photoreceptor precursors. This idea is consistent with the observation that the smaller number of LacZ-positive cells were observed in the retina in which the LacZ gene was directed by EELPOT compared with the number of LacZ-positive cells observed in the retina in which $L a c Z$ gene expression was directed by Otx2 BAC \#2. In addition, RAX did not bind to EELPOT in the P0 retina, supporting the observation that EELPOT is not active in the $\mathrm{P} 0$ retina.

The Otx2 expression in photoreceptor cells is significantly downregulated after birth when retinal bipolar cells begin ex- 
pressing Otx2 in the postnatal retina. The mechanism that downregulates Otx2 expression in photoreceptor cells after birth remains unclear.

Based on our analysis of the EELPOT enhancer, we propose a hypothetical model of the mechanisms of Otx2 expression that directs embryonic photoreceptor cell fate determination (Fig. 10). In proliferating RPCs, activated-NOTCH signaling represses Otx2 expression through HES family molecule-mediated suppression of the EELPOT enhancer, although RAX homeoprotein is expressed in proliferating RPCs. During the final cell cycle, the cell-extrinsic signal activating NOTCH turns off, leading to both the end of cell cycling and the removal of EELPOT suppression by HES. Then RAX is able to transactivate EELPOT resulting in Otx2 transactivation. Our model also may explain how the timing of cell fate determination occurs in the final cell cycle.

It remains unclear whether EELPOT is involved in rod cell fate determination. A different regulatory region(s) from EELPOT may be involved in activating Otx2 transcription in rod cell fate determination. Alternatively, although the EELPOT enhancer becomes very weak after $\mathrm{P} 0$, a low level of Otx2 expression may be sufficient for rod cell fate determination. Our observation that EELPOT-driven Otx2 expression restored not only the cone marker but also rhodopsin expression in the Otx2 CKO retina may support this idea. Extensive future analysis of an Otx2 enhancer region at postnatal stages might provide a definitive answer.

\section{Regulation of Otx2 transcription in RPCs by RAX}

Rax is a homeodomain-containing transcription factor expressed in RPCs, developing photoreceptor cells, and developing Müller glial cells in the vertebrate retina (Furukawa et al., 1997, 2000; Mathers et al., 1997; Chuang et al., 1999; Nelson et al., 2009; Pan et al., 2010). Since RAX is essential for RPC proliferation, we suppose that ectopic Pax6-positive cells observed in the Rax iCKO retina are amacrine-like cells rather than RPCs (Fig. $7 \mathrm{~V}, W)$, which are similar to the ones we reported in the Otx2 CKO retina (Nishida et al., 2003). These observations suggest that RAX plays multiple roles, including regulation of progenitor proliferation and cell differentiation, during retinal development. In chick, there are two Rax-like molecules, Rax and RaxL. RaxL, a Rax paralog in chicken, has been implicated in cone photoreceptor differentiation (Chen and Cepko, 2002), while Rax was reported to be involved in progenitor proliferation. No mouse ortholog of chicken RaxL has been identified, raising the possibility that mouse Rax might play roles both in progenitor proliferation and in cone development in mammals. However, it has not been investigated whether RAX regulates Otx2 transcription. Our results suggest that RAX acts as a direct activator of Otx2 transcription in RPCs. We showed that RAX can transactivate EELPOT in cultured cells. Moreover, through ChIP analysis we showed that RAX protein directly binds to EELPOT in the embryonic retina. We also observed that Otx2 expression was reduced in the RaxiCKO retina, indicating that RAX is essential for Otx2 transactivation in the embryonic retina.

We recently reported that the absence of BLIMP1, an OTX2 downstream transcription factor, led to an aberrant increase of Chx10 expression in developing photoreceptors (Katoh et al., 2010). In the Rax iCKO retina, Chx10 might be upregulated due to a defect of the OTX2-BLIMP1 pathway.

Rax has been implicated in the cell fate determination of Müller glia cells in the postnatal retina (Furukawa et al., 2000). However, it is unclear how Rax regulates both cone and Müller glia fate determination. Cell-intrinsic changes of RPC competence during retinal development may be associated with this mechanism. RAX function in cell fate determination may vary in a RPC competence-dependent manner.

\section{Negative regulation of $\mathrm{Otx} 2$ by NOTCH-HES signaling}

NOTCH signaling has been implicated in retinal cell differentiation (Cayouette and Raff, 2003; Baye and Link, 2007; Del Bene et al., 2008). It was recently reported that NOTCH1 inhibits photoreceptor production in the mammalian retina and that targeted disruption of Notch1 in the developing retina resulted in the enhanced expression of not only proneural genes like NeuroD1 and Math3, but also Otx2 and Crx, which are expressed exclusively in the photoreceptor cells in the embryonic retina at E13.5, suggesting that NOTCH signaling is involved in photoreceptor cell fate determination (Jadhav et al., 2006; Yaron et al., 2006). However, it has not been determined whether Otx2 expression was directly regulated by $\mathrm{NOTCH}$ signaling or not, because blocking $\mathrm{NOTCH}$ signaling induces neuronal differentiation in RPCs whereby transcription factors such as OTX2 are induced independently of NOTCH signaling as an indirect effect. Otx2 expression is upregulated in the $R B P j$-null retina, suggesting that $R B P j$-regulated genes, including Hes and Hey genes, are involved in Otx2 gene regulation. HES1 or HES5 single knock-out mice did not exhibit overproduction of photoreceptors (Hojo et al., 2000; Takatsuka et al., 2004; Riesenberg et al., 2009), suggesting that HES and/or HEY proteins are functionally redundant in repressing Otx2 transcription. Our ChIP assay results suggest that HES1, at least, binds to EELPOT. Together, our results suggest that N-box binding proteins, including HES or HEY family proteins, are involved in $O t x 2$ regulation directly in RPCs, serving a permissive role in Otx2 transcription. Since RAX, a transcriptional activator, is expressed in OTX2-positive photoreceptor precursors and HES1 expression, a transcriptional repressor, does not overlap with OTX2-positive cells, we suppose that HES family proteins may function as permissive factors, although we cannot exclude the possibility that NOTCH signaling may have an instructive role rather than a permissive role in photoreceptor cell fate determination. Our current study on EELPOT is consistent with various reports that $\mathrm{NOTCH}$ signaling has a role in photoreceptor cell fate determination (Jadhav et al., 2006; Yaron et al., 2006). Our results also suggest that HES and/or HEY proteins may regulate photoreceptor production, at least in part, by directly controlling Otx2 transcription machinery, although the molecular mechanisms that regulate the timing of $\mathrm{NOTCH}$ downregulation remain unknown. A cell-extrinsic signal is likely to regulate the NOTCH-RBPJ-HES pathway; however, understanding exactly what and how extrinsic signals regulate the NOTCH pathway in photoreceptor cell fate determination will await future study.

Our current study suggests that photoreceptor cell fate determination is realized, at least in part, through the activities of both cell intrinsic (RAX homeoprotein) and extrinsic (NOTCH-HES family) factors on a key enhancer locus (EELPOT) during the final cell cycle.

\section{References}

Akagi T, Inoue T, Miyoshi G, Bessho Y, Takahashi M, Lee JE, Guillemot F, Kageyama R (2004) Requirement of multiple basic helix-loop-helix genes for retinal neuronal subtype specification. J Biol Chem 279:28492-28498.

Alexiades MR, Cepko C (1996) Quantitative analysis of proliferation and cell cycle length during development of the rat retina. Dev Dyn 205:293-307.

Baye LM, Link BA (2007) Interkinetic nuclear migration and the selection of 
neurogenic cell divisions during vertebrate retinogenesis. J Neurosci 27:10143-10152.

Cayouette M, Raff M (2003) The orientation of cell division influences cell-fate choice in the developing mammalian retina. Development 130:2329-2339.

Chen CM, Cepko CL (2002) The chicken RaxL gene plays a role in the initiation of photoreceptor differentiation. Development 129:5363-5375.

Chuang JC, Mathers PH, Raymond PA (1999) Expression of three Rx homeobox genes in embryonic and adult zebrafish. Mech Dev 84:195-198.

Del Bene F, Wehman AM, Link BA, Baier H (2008) Regulation of neurogenesis by interkinetic nuclear migration through an apical-basal notch gradient. Cell 134:1055-1065.

Deng C, Wynshaw-Boris A, Zhou F, Kuo A, Leder P (1996) Fibroblast growth factor receptor 3 is a negative regulator of bone growth. Cell 84:911-921.

Dyer MA, Livesey FJ, Cepko CL, Oliver G (2003) Prox1 function controls progenitor cell proliferation and horizontal cell genesis in the mammalian retina. Nat Genet 34:53-58.

Feil R, Wagner J, Metzger D, Chambon P (1997) Regulation of Cre recombinase activity by mutated estrogen receptor ligand-binding domains. Biochem Biophys Res Commun 237:752-757.

Fischer A, Gessler M (2007) Delta-Notch-and then? Protein interactions and proposed modes of repression by Hes and Hey bHLH factors. Nucleic Acids Res 35:4583-4596.

Furukawa A, Koike C, Lippincott P, Cepko CL, Furukawa T (2002) The mouse Crx $5^{\prime}$-upstream transgene sequence directs cell-specific and developmentally regulated expression in retinal photoreceptor cells. J Neurosci 22:1640-1647.

Furukawa T, Kozak CA, Cepko CL (1997) rax, a novel paired-type homeobox gene, shows expression in the anterior neural fold and developing retina. Proc Natl Acad Sci U S A 94:3088-3093.

Furukawa T, Mukherjee S, Bao ZZ, Morrow EM, Cepko CL (2000) rax, Hes 1, and notch1 promote the formation of Muller glia by postnatal retinal progenitor cells. Neuron 26:383-394.

Gong S, Yang XW, Li C, Heintz N (2002) Highly efficient modification of bacterial artificial chromosomes (BACs) using novel shuttle vectors containing the R6Kgamma origin of replication. Genome Res 12:1992-1998.

Hojo M, Ohtsuka T, Hashimoto N, Gradwohl G, Guillemot F, Kageyama R (2000) Glial cell fate specification modulated by the bHLH gene Hes5 in mouse retina. Development 127:2515-2522.

Holt CE, Bertsch TW, Ellis HM, Harris WA (1988) Cellular determination in the Xenopus retina is independent of lineage and birth date. Neuron 1:15-26.

Hufnagel RB, Le TT, Riesenberg AL, Brown NL (2010) Neurog2 controls the leading edge of neurogenesis in the mammalian retina. Dev Biol 340:490-503.

Iso T, Kedes L, Hamamori Y (2003) HES and HERP families: multiple effectors of the Notch signaling pathway. J Cell Physiol 194:237-255.

Jadhav AP, Mason HA, Cepko CL (2006) Notch 1 inhibits photoreceptor production in the developing mammalian retina. Development 133:913-923.

Katoh K, Omori Y, Onishi A, Sato S, Kondo M, Furukawa T (2010) Blimp1 suppresses Chx10 expression in differentiating retinal photoreceptor precursors to ensure proper photoreceptor development. J Neurosci 30:6515-6526.

Koike C, Nishida A, Ueno S, Saito H, Sanuki R, Sato S, Furukawa A, Aizawa S, Matsuo I, Suzuki N, Kondo M, Furukawa T (2007) Functional roles of Otx2 transcription factor in postnatal mouse retinal development. Mol Cell Biol 27:8318-8329.

Kothary R, Clapoff S, Darling S, Perry MD, Moran LA, Rossant J (1989) Inducible expression of an hsp68-lac $Z$ hybrid gene in transgenic mice. Development 105:707-714.

Kurokawa D, Takasaki N, Kiyonari H, Nakayama R, Kimura-Yoshida C, Matsuo I, Aizawa S (2004a) Regulation of Otx2 expression and its functions in mouse epiblast and anterior neuroectoderm. Development 131:3307-3317.

Kurokawa D, Kiyonari H, Nakayama R, Kimura-Yoshida C, Matsuo I, Aizawa S (2004b) Regulation of Otx2 expression and its functions in mouse forebrain and midbrain. Development 131:3319-3331.

Levine EM, Green ES (2004) Cell-intrinsic regulators of proliferation in vertebrate retinal progenitors. Semin Cell Dev Biol 15:63-74.

Livesey FJ, Cepko CL (2001) Vertebrate neural cell-fate determination: lessons from the retina. Nat Rev Neurosci 2:109-118.
Mathers PH, Grinberg A, Mahon KA, Jamrich M (1997) The Rx homeobox gene is essential for vertebrate eye development. Nature 387:603-607.

McConnell SK, Kaznowski CE (1991) Cell cycle dependence of laminar determination in developing neocortex. Science 254:282-285.

Muranishi Y, Sato S, Inoue T, Ueno S, Koyasu T, Kondo M, Furukawa T (2010) Gene expression analysis of embryonic photoreceptor precursor cells using BAC-Crx-EGFP transgenic mouse. Biochem Biophys Res Commun 392:317-322.

Nelson SM, Park L, Stenkamp DL (2009) Retinal homeobox 1 is required for retinal neurogenesis and photoreceptor differentiation in embryonic zebrafish. Dev Biol 328:24-39.

Nishida A, Furukawa A, Koike C, Tano Y, Aizawa S, Matsuo I, Furukawa T (2003) Otx2 homeobox gene controls retinal photoreceptor cell fate and pineal gland development. Nat Neurosci 6:1255-1263.

Ohsawa R, Kageyama R (2008) Regulation of retinal cell fate specification by multiple transcription factors. Brain Res 1192:90-98.

Pan Y, Martinez-De Luna RI, Lou CH, Nekkalapudi S, Kelly LE, Sater AK, El-Hodiri HM (2010) Regulation of photoreceptor gene expression by the retinal homeobox $(R x)$ gene product. Dev Biol 339:494-506.

Riesenberg AN, Liu Z, Kopan R, Brown NL (2009) Rbpj cell autonomous regulation of retinal ganglion cell and cone photoreceptor fates in the mouse retina. J Neurosci 29:12865-12877.

Sakai K, Miyazaki J (1997) A transgenic mouse line that retains Cre recombinase activity in mature oocytes irrespective of the cre transgene transmission. Biochem Biophys Res Commun 237:318-324.

Sanuki R, Omori Y, Koike C, Sato S, Furukawa T (2010) Panky, a novel photoreceptor-specific ankyrin repeat protein, is a transcriptional cofactor that suppresses CRX-regulated photoreceptor genes. FEBS Lett 584:753-758.

Sato S, Inoue T, Terada K, Matsuo I, Aizawa S, Tano Y, Fujikado T, Furukawa $\mathrm{T}$ (2007) Dkk3-Cre BAC transgenic mouse line: a tool for highly efficient gene deletion in retinal progenitor cells. Genesis 45:502-507.

Sato S, Omori Y, Katoh K, Kondo M, Kanagawa M, Miyata K, Funabiki K, Koyasu T, Kajimura N, Miyoshi T, Sawai H, Kobayashi K, Tani A, Toda T, Usukura J, Tano Y, Fujikado T, Furukawa T (2008) Pikachurin, a dystroglycan ligand, is essential for photoreceptor ribbon synapse formation. Nat Neurosci 11:923-931.

Shang Y, Hu X, DiRenzo J, Lazar MA, Brown M (2000) Cofactor dynamics and sufficiency in estrogen receptor-regulated transcription. Cell 103:843-852.

Takatsuka K, Hatakeyama J, Bessho Y, Kageyama R (2004) Roles of the bHLH gene Hes1 in retinal morphogenesis. Brain Res 1004:148-155.

Tian E, Kimura C, Takeda N, Aizawa S, Matsuo I (2002) Otx2 is required to respond to signals from anterior neural ridge for forebrain specification. Dev Biol 242:204-223.

Trimarchi JM, Stadler MB, Cepko CL (2008) Individual retinal progenitor cells display extensive heterogeneity of gene expression. PLoS One 3:e1588.

Turner DL, Cepko CL (1987) A common progenitor for neurons and glia persists in rat retina late in development. Nature 328:131-136.

Turner DL, Snyder EY, Cepko CL (1990) Lineage-independent determination of cell type in the embryonic mouse retina. Neuron 4:833-845.

Walsh C, Cepko CL (1988) Clonally related cortical cells show several migration patterns. Science 241:1342-1345.

Walsh C, Cepko CL (1993) Clonal dispersion in proliferative layers of developing cerebral cortex. Nature 362:632-635.

Wetts R, Fraser SE (1988) Multipotent precursors can give rise to all major cell types of the frog retina. Science 239:1142-1145.

Wilson D, Sheng G, Lecuit T, Dostatni N, Desplan C (1993) Cooperative dimerization of paired class homeo domains on DNA. Genes Dev 7:2120-2134.

Yang XW, Model P, Heintz N (1997) Homologous recombination based modification in Escherichia coli and germline transmission in transgenic mice of a bacterial artificial chromosome. Nat Biotechnol 15:859-865.

Yaron O, Farhy C, Marquardt T, Applebury M, Ashery-Padan R (2006) Notch1 functions to suppress cone-photoreceptor fate specification in the developing mouse retina. Development 133:1367-1378.

Zheng MH, Shi M, Pei Z, Gao F, Han H, Ding YQ (2009) The transcription factor RBP-J is essential for retinal cell differentiation and lamination. Mol Brain 2:38. 